ANALYSIS \& PDE Volume $6 \quad$ No. $8 \quad 2013$

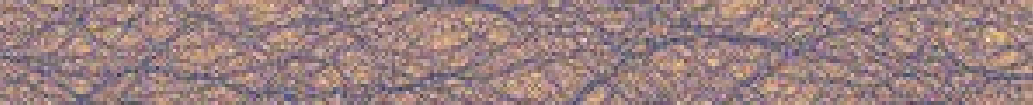
LONGZHI LIN

UNIFORMITY OF HARMONIC MAP HEAT FLOW AT INFINITE TIME 


\title{
UNIFORMITY OF HARMONIC MAP HEAT FLOW AT INFINITE TIME
}

\author{
LONGZHI LIN
}

We show an energy convexity along any harmonic map heat flow with small initial energy and fixed boundary data on the unit 2-disk. In particular, this gives an affirmative answer to a question raised by W. Minicozzi asking whether such harmonic map heat flow converges uniformly in time strongly in the $W^{1,2}$-topology, as time goes to infinity, to the unique limiting harmonic map.

\section{Introduction}

Given a compact Riemannian manifold $\mu$ and a closed (that is, compact and without boundary) Riemannian manifold $\mathcal{N}$ which is an isometrically embedded submanifold of $\mathbb{R}^{n}$, we can define the Dirichlet energy of a map $u \in W^{1,2}(\mathcal{M}, \mathcal{N})$ :

$$
\operatorname{Energy}(u)=E(u)=\frac{1}{2} \int_{\mathcal{M}}|\nabla u|^{2} d v_{\mathcal{M}}
$$

where $W^{1,2}(\mathcal{M}, \mathcal{N})$ is the class of maps

$$
\left\{u \in L_{\text {loc }}^{1}\left(M, \mathbb{R}^{n}\right): \int_{\mathcal{M}}|\nabla u|^{2} d v_{\mathcal{M}}<+\infty, u(x) \in \mathcal{N} \text { for a.e. } x \in \mathcal{M}\right\} .
$$

The tension field $\tau(u) \in \Gamma\left(u^{*}(T \mathcal{N})\right)$ is the vector field along $u$ representing the negative $L^{2}$-gradient of $E(u)$. A weakly harmonic map $u$ from $M$ to $\mathcal{N}$ is a critical point of the energy functional $E(u)$ in the distribution sense, that is, the tension field $\tau(u)$ vanishes, and it solves the Euler-Lagrange equation

$$
-\Delta_{\mu} u=\Pi(u)(\nabla u, \nabla u)
$$

where $u=\left(u^{1}, \ldots, u^{n}\right)$ and $\Pi(u)$ denotes the second fundamental form of $\mathcal{N} \hookrightarrow \mathbb{R}^{n}$ at the point $u$. We refer to this system of elliptic equations as the harmonic map equation.

A natural way to control the tension field for an energy minimizing sequence of maps and to get the existence of harmonic maps from $\mathcal{M}$ to $\mathcal{N}$ is to consider the initial (-boundary) value problem:

$$
\begin{cases}u_{t}-\Delta_{\mathcal{M}} u=\Pi(u)(\nabla u, \nabla u) & \text { on } \mathcal{M} \times(0, T), \\ u(x, 0)=u_{0}(x) & \text { for } x \in \mathcal{M}, \\ u(x, t)=\chi(x)=\left.u_{0}\right|_{\partial \mathcal{M}} & \text { for all } t \geq 0, x \in \partial \mathcal{M} \text { if } \partial \mathcal{M} \neq \varnothing,\end{cases}
$$

MSC2010: 53C44, 58E20.

Keywords: harmonic map heat flow, energy convexity, uniform convergence. 
where $u=\left(u^{1}, \ldots, u^{n}\right)$ and $T>0$. We refer to this system of parabolic equations as the harmonic map heat flow, to the map $u_{0}$ as the initial data, and to the map $\chi$ as the boundary data. Given $u_{0} \in W^{1,2}(\mathcal{M}, \mathcal{N})$ and $\chi=\left.u_{0}\right|_{\partial \mathcal{M}} \in W^{1 / 2,2}(\partial \mathcal{M}, \mathcal{N})$, we define $u \in W^{1,2}(\mathcal{M} \times[0, T], \mathcal{N})$ to be the weak solution of (1-3) if

$$
\int_{0}^{T} \int_{\mathcal{M}}\left\langle u_{t}, \xi\right\rangle+\langle\nabla u, \nabla \xi\rangle-\langle\Pi(u)(\nabla u, \nabla u), \xi\rangle d x d t=0
$$

for any $\xi \in C_{c}^{\infty}\left(\mathcal{M} \times(0, T), \mathbb{R}^{n}\right)$.

In the fundamental paper where the harmonic map heat flow was first introduced, Eells and Sampson [1964] proved that the harmonic map heat flow exists for all time in the case where the source domain $\mathcal{M}$ (of arbitrary dimensions) is without boundary and the target manifold $\mathcal{N}$ has nonpositive sectional curvature. They also proved that there exists some sequence of times $t_{i} \nearrow+\infty$ such that

$$
u_{\infty}=\lim _{i \rightarrow \infty} u\left(\cdot, t_{i}\right)
$$

is a harmonic map from $\mu$ to $\mathcal{N}$. The case in which the source domain $M$ has boundary was dealt with in [Hamilton 1975] under the same curvature assumption on $\mathcal{N}$. The question of uniformity of the convergence in time of the flow considered by Eells and Sampson was left open at that stage, but it was settled later by Hartman. We shall state their results in the following theorem.

Theorem 1.1 [Eells and Sampson 1964; Hartman 1967]. Suppose that $\mathcal{M}$ and $\mathcal{N}$ are two closed Riemannian manifolds and that $\mathcal{N}$ has nonpositive sectional curvature. Then, given any $u_{0} \in C^{1}(\mathcal{M}, \mathcal{N})$, the harmonic map heat flow has a unique solution $u \in C^{1}(\mathcal{M} \times[0, \infty), \mathcal{N}) \cap C^{\infty}(\mathcal{M} \times(0, \infty), \mathcal{N})$. Moreover,

$$
u_{\infty}=\lim _{t \rightarrow \infty} u(\cdot, t)
$$

exists uniformly in $C^{k}$-topology for all $k \geq 0$ and $u_{\infty}$ is a harmonic map homotopic to $u_{0}$.

Other similar uniformity results were obtainable under various assumptions on the target manifold $\mathcal{N}$, such as being real analytic [Simon 1983] or admitting a strictly convex function; see also the interesting paper [Topping 1997] for harmonic map heat flow in a special case in which both the source and target manifolds are 2-spheres $\mathbb{S}^{2}$.

When the dimension of the source domain $\mathcal{M}$ is two, things are particularly interesting because the energy functional $E(u)$ and the harmonic map equation (1-2) are conformally invariant in this critical dimension. Regarding the harmonic map heat flow (1-3) from surfaces to a general closed target manifold $\mathcal{N}$, the first fundamental work was [Struwe 1985], dealing with the case $\partial \mathcal{M}=\varnothing$, where "bubbles" may occur and have been analyzed in detail. This result was then extended to the case $\partial \mathcal{M} \neq \varnothing$ with Dirichlet boundary condition in [Chang 1989]. If the initial energy $E\left(u_{0}\right)$ is sufficiently small, it is well known by now that the weak solution of (1-3) is smooth (in the interior) by the results of [Freire 1995; 1996] using the so-called moving frame technique introduced by Hélein (see, for example, [Hélein 2002]). We will state their $\varepsilon$-regularity theorem required in this paper, and include an alternative proof of it for self-containedness; it uses the main tool of our current work, which we call Rivière's gauge decomposition (Theorem 3.7). 
Theorem 1.2 ([Freire 1995; 1996]; cf. [Struwe 1985; Chang 1989; Wang 2012]). Let $M$ be a simply connected compact Riemannian surface and $\mathcal{N}$ a closed Riemannian manifold. There exists $\varepsilon_{0}>0$ depending only on $\mathcal{M}$ and $\mathcal{N}$ such that the following is true. For each initial data $u_{0} \in W^{1,2}(\mathcal{M}, \mathcal{N})$ with $E\left(u_{0}\right)<\varepsilon_{0}$ and the boundary data $\chi=\left.u_{0}\right|_{\partial \mu}$ in the case where $\partial \mathcal{M} \neq \varnothing$, there exists a unique global weak solution $u \in W^{1,2}(\mathcal{M} \times[0, \infty), \mathcal{N})$ for which $E(u(\cdot, t))$ is nonincreasing in $t$. Also, $u$ is smooth in $M \times[1, \infty)$ and, for any $t_{2}>t_{1} \geq 1$, we have

$$
2 \int_{t_{1}}^{t_{2}} \int_{\mathcal{M}}\left|u_{t}\right|^{2}=\int_{B_{1}}\left|\nabla u\left(\cdot, t_{1}\right)\right|^{2}-\int_{B_{1}}\left|\nabla u\left(\cdot, t_{2}\right)\right|^{2} .
$$

Moreover, there exists some sequence of times $t_{i} \nearrow+\infty$ such that

$$
u_{\infty}=\lim _{i \rightarrow \infty} u\left(\cdot, t_{i}\right)
$$

exists in the $C^{k}$-topology for any $k \geq 0$ and $u_{\infty}$ is a harmonic map from $\mathcal{M}$ to $\mathcal{N}$.

Remark 1.3. In particular, in order to avoid the "bubble" (singularity) along the harmonic map heat flow, a priori we may choose $\varepsilon_{0}<K_{1}+K_{2}$ where

$$
K_{1}=\inf \left\{E(v) \mid v \in W^{1,2}(\mathcal{M}, \mathcal{N}) \text { and }\left.v\right|_{\partial \mathcal{M}}=\chi\right\}
$$

and

$$
K_{2}=\inf \left\{E(v) \mid v: \mathbb{S}^{2} \rightarrow \mathcal{N} \text { is nonconstant and harmonic }\right\}>0 .
$$

Remark 1.4. Freire's regularity results for harmonic map heat flow represent a parabolic version of the regularity theorem of Hélein stating that weakly harmonic maps from surfaces are regular; see, for example, [Hélein 2002].

A tempting question to ask is whether, for a general closed target manifold $\mathcal{N}$ (without additional geometric assumptions), one could establish uniformity results for the harmonic map heat flow similar to Theorem 1.1. In particular, is the convergence (1-7) in Theorem 1.2 uniform for all time in the natural $W^{1,2}$-topology, say? In view of the conformal invariance of the energy functional $E(u)$ in dimension two, the condition of small energy seems to be a natural candidate to work with in order to get such uniformity of the convergence in time for the flow. We will show in the following that this is indeed the case. In what follows we will concentrate on the case where the source domain $M$ is a simply connected compact Riemannian surface with boundary. More precisely, we focus on domains which are conformally equivalent to the unit 2-disk $B_{1} \subset \mathbb{R}^{2}$. From now on we will only work on $B_{1}$ :

$$
\begin{cases}u_{t}-\Delta u=\Pi(u)(\nabla u, \nabla u) & \text { on } B_{1} \times(0, T), \\ u(x, 0)=u_{0}(x) & \text { for } x \in B_{1}, \\ u(x, t)=\chi(x)=\left.u_{0}\right|_{\partial B_{1}} & \text { for all } t \geq 0 \text { and } x \in \partial B_{1},\end{cases}
$$

where $\Delta$ is the usual Laplacian $\Delta=\sum_{i=1}^{2} \partial^{2} / \partial x_{i}^{2}$ in $\mathbb{R}^{2}$. All the arguments could be easily modified to apply to the general case.

Notation 1.5. In what follows, $\nabla=\left(\partial_{x}, \partial_{y}\right)$ is the gradient operator in $\mathbb{R}^{2}$ and $\nabla^{\perp}=\left(-\partial_{y}, \partial_{x}\right)$ denotes the orthogonal gradient (that is, $\nabla^{\perp}$ is the $\nabla$-operator rotated by $\pi / 2$ ). 
Now we state the main theorem of this paper.

Theorem 1.6. Let $\mathcal{N}$ be a closed Riemannian manifold. There exist $\varepsilon_{0}, T_{0}>0$ depending only on $\mathcal{N}$ such that if $u \in W^{1,2}\left(B_{1} \times[0, \infty), \mathcal{N}\right)$ is a global weak solution to the harmonic map heat flow (1-8) with $E\left(u_{0}\right)<\varepsilon_{0}, E(u(\cdot, t))$ is nonincreasing in $t$, and $\left.u(\cdot, t)\right|_{\partial B_{1}}=\chi$ for all $t \geq 0$, then, for all $t_{2}>t_{1} \geq T_{0}$, we have the energy convexity

$$
\frac{1}{4} \int_{B_{1}}\left|\nabla u\left(\cdot, t_{1}\right)-\nabla u\left(\cdot, t_{2}\right)\right|^{2} \leq \int_{B_{1}}\left|\nabla u\left(\cdot, t_{1}\right)\right|^{2}-\int_{B_{1}}\left|\nabla u\left(\cdot, t_{2}\right)\right|^{2} .
$$

Remark 1.7. We do not know if the energy convexity (1-9) holds for all $t_{2}>t_{1} \geq 0$. In the following arguments we agree to let $\varepsilon_{0}$ be sufficiently small and $T_{0}$ be sufficiently large, as needed.

Our approach to the proof of Theorem 1.6 is based on the technique we call Rivière's gauge decomposition, introduced in [Rivière 2007]; see Section 3. Immediate applications of Theorem 1.6 are:

Corollary 1.8. Let $\mathcal{N}$ be a closed Riemannian manifold. There exists $\varepsilon_{0}>0$ depending only on $\mathcal{N}$ such that if $u \in W^{1,2}\left(B_{1} \times[0, \infty), \mathcal{N}\right)$ is a global weak solution to the harmonic map heat flow (1-8) with $E\left(u_{0}\right)<\varepsilon_{0}, E(u(\cdot, t))$ is nonincreasing in $t$, and $\left.u(\cdot, t)\right|_{\partial B_{1}}=\chi$ for all $t \geq 0$, then

$$
u(\cdot, t) \rightarrow u_{\infty} \quad \text { uniformly as } t \rightarrow+\infty \quad \text { strongly in } W^{1,2}\left(B_{1}, \mathbb{R}^{n}\right),
$$

where $u_{\infty}$ is the unique harmonic map with $E\left(u_{\infty}\right)<\varepsilon_{0}$ and boundary data $\chi$.

Corollary 1.9. Let $M$ be a two dimensional domain that is conformally equivalent to $B_{1}$ and has smooth boundary, and let $\mathcal{N}$ be a closed Riemannian manifold. Suppose the initial energy $E\left(u_{0}\right)<\varepsilon_{0}$. Then the harmonic map heat flow (1-3) with initial data $u_{0} \in C^{2, \alpha}(\overline{\mathcal{M}}, \mathcal{N})$ and boundary data $\chi \in C^{2, \alpha}(\partial \mathcal{M}, \mathcal{N})$, considered by Chang [1989], converges uniformly in time strongly in $W^{1,2}(\mathcal{M}, \mathcal{N})$ to the unique harmonic map $u_{\infty} \in C_{\chi}^{2, \alpha}(\bar{M}, \mathcal{N})$.

Remark 1.10. We do not know if a harmonic map heat flow can be nonuniform without the small energy assumption. In view of the nonuniqueness results of Brezis and Coron [1983] and Jost [1984] for harmonic maps (with large energy) sharing the same boundary data on $\partial B_{1}$, it is quite possible that the small energy assumption is necessary for the energy convexity and uniform convergence of the flow in Theorem 1.6 and Corollary 1.8 to hold.

Remark 1.11. Colding and Minicozzi [2008a] showed an energy convexity for weakly harmonic maps with small energy on $B_{1}$ : there exists $\varepsilon_{0}>0$ such that if $u, v \in W^{1,2}\left(B_{1}, \mathcal{N}\right)$ with $\left.u\right|_{\partial B_{1}}=\left.v\right|_{\partial B_{1}}, E(u)<\varepsilon_{0}$, and $u$ is weakly harmonic, then we have the energy convexity

$$
\frac{1}{2} \int_{B_{1}}|\nabla v-\nabla u|^{2} \leq \int_{B_{1}}|\nabla v|^{2}-\int_{B_{1}}|\nabla u|^{2} .
$$

See [Lamm and Lin 2013] for an alternative proof of this energy convexity using the same techniques used in the present paper. A direct consequence of (1-11) is that $u_{\infty}$ in Corollary 1.8 is unique in the class

$$
\left\{v \in W^{1,2}\left(B_{1}, \mathbb{R}^{n}\right): E(v)<\varepsilon_{0} \text { and }\left.v\right|_{\partial B_{1}}=\chi\right\} ;
$$

see [Colding and Minicozzi 2008a, Corollary 3.3]. 
The paper is organized as follows. In Section 2 we present some heuristic arguments and elaborate on the idea of the proof of the main theorem, Theorem 1.6. In Section 3 we review the main tool of our proof, namely, Rivière's gauge decomposition technique adapted to the case of harmonic map heat flow. In Section 4 we show improved estimates for Rivière's matrices $B$ and $P$, which are the two key ingredients of our proof. We finish the proof of our main theorem in Section 5.

\section{Heuristic arguments and the idea of the proof}

In this section we will present some heuristic arguments and sketch the basic idea of the proof of Theorem 1.6. We will abbreviate $u(\cdot, t)$ to $u(t)$. In order to prove the energy convexity (1-9) along the harmonic map heat flow, that is, there exists some $T_{0}>0$ such that, for all $t_{2}>t_{1} \geq T_{0}$, we have

$$
\frac{1}{4} \int_{B_{1}}\left|\nabla u\left(\cdot, t_{1}\right)-\nabla u\left(\cdot, t_{2}\right)\right|^{2} \leq \int_{B_{1}}\left|\nabla u\left(\cdot, t_{1}\right)\right|^{2}-\int_{B_{1}}\left|\nabla u\left(\cdot, t_{2}\right)\right|^{2},
$$

it suffices to show

$$
\Psi \geq-\left(\int_{B_{1}}\left|\nabla u\left(t_{1}\right)\right|^{2}-\int_{B_{1}}\left|\nabla u\left(t_{2}\right)\right|^{2}\right)-\frac{1}{2} \int_{B_{1}}\left|\nabla u\left(t_{1}\right)-\nabla u\left(t_{2}\right)\right|^{2},
$$

where (using that $\left.u(\cdot, t)\right|_{\partial B_{1}}=\chi$ for all $t \geq 0$ and the flow equation (1-8))

$$
\begin{aligned}
\Psi & :=\int_{B_{1}}\left|\nabla u\left(t_{1}\right)\right|^{2}-\int_{B_{1}}\left|\nabla u\left(t_{2}\right)\right|^{2}-\int_{B_{1}}\left|\nabla u\left(t_{1}\right)-\nabla u\left(t_{2}\right)\right|^{2} \\
& =2 \int_{B_{1}}\left\langle\nabla u\left(t_{1}\right)-\nabla u\left(t_{2}\right), \nabla u\left(t_{2}\right)\right\rangle \\
& =-2 \int_{B_{1}}\left\langle u\left(t_{1}\right)-u\left(t_{2}\right), u_{t}\left(t_{2}\right)-\Pi(u)(\nabla u, \nabla u)\left(t_{2}\right)\right\rangle .
\end{aligned}
$$

Now note that for any $p, q \in \mathcal{N}$, there exists some constant $C>0$ depending only on $\mathcal{N}$ such that $\left|(p-q)^{\perp}\right| \leq C|p-q|^{2}$, where the superscript $\perp$ denotes the normal component of a vector; see, for example, [Colding and Minicozzi 2008b, Lemma A.1]. Therefore, using the fact that $\Pi(u)(\nabla u, \nabla u) \perp T_{u} \mathcal{N}$ and the Cauchy-Schwarz inequality, (2-3) yields

$$
\begin{aligned}
\Psi & \geq-2\left(\int_{B_{1}}\left|u\left(t_{1}\right)-u\left(t_{2}\right)\right|^{2}\right)^{1 / 2}\left(\int_{B_{1}}\left|u_{t}\left(t_{2}\right)\right|^{2}\right)^{1 / 2}-C \int_{B_{1}}\left|\left(u\left(t_{1}\right)-u\left(t_{2}\right)\right)^{\perp}\right|\left|\nabla u\left(t_{2}\right)\right|^{2} \\
& \geq-2 \sqrt{t_{2}-t_{1}}\left(\int_{t_{1}}^{t_{2}} \int_{B_{1}}\left|u_{t}\right|^{2}\right)^{1 / 2}\left(\int_{B_{1}}\left|u_{t}\left(t_{2}\right)\right|^{2}\right)^{1 / 2}-C \int_{B_{1}}\left|u\left(t_{1}\right)-u\left(t_{2}\right)\right|^{2}\left|\nabla u\left(t_{2}\right)\right|^{2},
\end{aligned}
$$

where we also used the smoothness and compactness of the target manifold $\mathcal{N}$. Here and throughout the rest of the paper, $C>0$ will denote a universal constant depending only on $\mathcal{N}$ unless otherwise stated.

Since we have (1-6) and $\varepsilon_{0}$ can always be chosen sufficiently small, we know that (2-2) will be achieved if we can show the following two key propositions. 
Proposition 2.1. Let $u(x, t)$ be as in Theorem 1.6. Then there exists $T_{0}>0$ such that, for all $t_{2}>t_{1} \geq T_{0}$, we have

$$
\int_{B_{1}}\left|u_{t}\left(t_{2}\right)\right|^{2} \leq \frac{1}{t_{2}-t_{1}} \int_{t_{1}}^{t_{2}} \int_{B_{1}}\left|u_{t}\right|^{2} .
$$

Remark 2.2. The key point of Proposition 2.1 is that (2-4) is valid for all $t_{2}>t_{1} \geq T_{0}$. We will see that, in fact, $\int_{B_{1}}\left|u_{t}(t)\right|^{2}$ is nonincreasing along the flow after $T_{0}$, which yields (2-4); cf. Lemma 2.5 and (5-16) below. A similar but weaker estimate was shown when the source domain of the heat flow is boundaryless [Struwe 1985, Equation (3.5)], which turned out to be the key estimate needed in Struwe's proof.

Proposition 2.3. Let $u(x, t)$ be as in Theorem 1.6. Then there exists $T_{0}>0$ such that, for all $t_{2}>t_{1} \geq T_{0}$, we have

$$
\int_{B_{1}}\left|u\left(t_{1}\right)-u\left(t_{2}\right)\right|^{2}\left|\nabla u\left(t_{2}\right)\right|^{2} \leq C \varepsilon_{0} \int_{B_{1}}\left|\nabla u\left(t_{1}\right)-\nabla u\left(t_{2}\right)\right|^{2} .
$$

If one were able to get

$$
\left\|\nabla u\left(t_{2}\right)\right\|_{L^{\infty}\left(B_{1}\right)} \leq C \sqrt{\varepsilon_{0}},
$$

(2-5) would have been automatically true by Poincaré's inequality. However, without imposing any regularity information on the boundary data $\chi$, it will be hopeless to get such a strong global pointwise gradient estimate. In fact, even if we look at the stationary case, that is, $W^{1,2}$-weakly harmonic maps on $B_{1}$, it is easy to convince oneself that it is unreasonable to expect regularity with global estimates on the whole $B_{1}$ better than $W^{2,2}$ in general.

Nevertheless, not all hope is lost to show estimates (2-4) and (2-5). Indeed, the following lemma is true, which validates Proposition 2.3 under some extra assumptions.

Lemma 2.4. Let $u(x, t)$ be as in Theorem 1.6 and suppose that, for all $t_{2}>t_{1} \geq T_{0} \geq 1$, we can solve the following Dirichlet problem for $\psi \in W_{0}^{1,2} \cap L^{\infty}\left(B_{1}\right)$ :

$$
\begin{cases}\Delta \psi=\left|\nabla u\left(t_{2}\right)\right|^{2} & \text { in } B_{1} \\ \psi=0 & \text { on } \partial B_{1}\end{cases}
$$

with the estimate

$$
\|\psi\|_{L^{\infty}\left(B_{1}\right)}+\|\nabla \psi\|_{L^{2}\left(B_{1}\right)} \leq C \varepsilon_{0}
$$

Then Proposition 2.3 holds.

Proof. The proof is essentially taken from [Colding and Minicozzi 2008a]. Substituting (2-7) into the left-hand side of (2-5) yields (using also that $u\left(t_{1}\right)=u\left(t_{2}\right)=\chi$ on $\partial B_{1}$ )

$$
\begin{aligned}
\int_{B_{1}}\left|u\left(t_{1}\right)-u\left(t_{2}\right)\right|^{2}\left|\nabla u\left(t_{2}\right)\right|^{2} & =\int_{B_{1}}\left|u\left(t_{1}\right)-u\left(t_{2}\right)\right|^{2} \Delta \psi \leq \int_{B_{1}}|\nabla| u\left(t_{1}\right)-\left.u\left(t_{2}\right)\right|^{2}|| \nabla \psi \mid \\
& \leq 2\left(\int_{B_{1}}\left|\nabla u\left(t_{1}\right)-\nabla u\left(t_{2}\right)\right|^{2}\right)^{1 / 2}\left(\int_{B_{1}}\left|u\left(t_{1}\right)-u\left(t_{2}\right)\right|^{2}|\nabla \psi|^{2}\right)^{1 / 2},
\end{aligned}
$$

where we have applied Stokes' theorem to $\operatorname{div}\left(\left|u\left(t_{1}\right)-u\left(t_{2}\right)\right|^{2} \nabla \psi\right)$ and used the Cauchy-Schwarz inequality. Now, applying Stokes' theorem to $\operatorname{div}\left(\left|u\left(t_{1}\right)-u\left(t_{2}\right)\right|^{2} \psi \nabla \psi\right)$ and using that $\Delta \psi \geq 0$ and (2-9), 
we have

$$
\begin{aligned}
\int_{B_{1}}\left|u\left(t_{1}\right)-u\left(t_{2}\right)\right|^{2}|\nabla \psi|^{2} & \leq \int_{B_{1}}|\psi|\left(\left|u\left(t_{1}\right)-u\left(t_{2}\right)\right|^{2} \Delta \psi+|\nabla| u\left(t_{1}\right)-\left.u\left(t_{2}\right)\right|^{2}|| \nabla \psi \mid\right) \\
& \leq 4\|\psi\|_{L^{\infty}}\left(\int_{B_{1}}\left|\nabla u\left(t_{1}\right)-\nabla u\left(t_{2}\right)\right|^{2}\right)^{1 / 2}\left(\int_{B_{1}}\left|u\left(t_{1}\right)-u\left(t_{2}\right)\right|^{2}|\nabla \psi|^{2}\right)^{1 / 2},
\end{aligned}
$$

so that

$$
\left(\int_{B_{1}}\left|u\left(t_{1}\right)-u\left(t_{2}\right)\right|^{2}|\nabla \psi|^{2}\right)^{1 / 2} \leq 4\|\psi\|_{L^{\infty}}\left(\int_{B_{1}}\left|\nabla u\left(t_{1}\right)-\nabla u\left(t_{2}\right)\right|^{2}\right)^{1 / 2} .
$$

Finally, substituting (2-11) back into (2-9) and combining with (2-8) (and choosing $\varepsilon_{0}$ sufficiently small), yields

$$
\int_{B_{1}}\left|u\left(t_{1}\right)-u\left(t_{2}\right)\right|^{2}\left|\nabla u\left(t_{2}\right)\right|^{2} \leq C\|\psi\|_{L^{\infty}} \int_{B_{1}}\left|\nabla u\left(t_{1}\right)-\nabla u\left(t_{2}\right)\right|^{2} \leq C \varepsilon_{0} \int_{B_{1}}\left|\nabla u\left(t_{1}\right)-\nabla u\left(t_{2}\right)\right|^{2},
$$

which is just (2-5).

Similarly, we can show the following lemma, which states, under some extra conditions, that $\int_{B_{1}}\left|u_{t}(t)\right|^{2}$ is nonincreasing along the harmonic map heat flow after some $T_{0}>0$ and Proposition 2.1 can be validated in this case.

Lemma 2.5. Let $u(x, t)$ be as in Theorem 1.6. For any $t_{2}>t_{1} \geq T_{0} \geq 1$, suppose that for any $t_{0} \in\left[t_{1}, t_{2}\right]$ we can solve the following Dirichlet problem for $\psi \in W_{0}^{1,2} \cap L^{\infty}\left(B_{1}\right)$ :

$$
\begin{cases}\Delta \psi=\left|\nabla u\left(t_{0}\right)\right|^{2} & \text { in } B_{1} \\ \psi=0 & \text { on } \partial B_{1}\end{cases}
$$

with the estimate

$$
\|\psi\|_{L^{\infty}\left(B_{1}\right)}+\|\nabla \psi\|_{L^{2}\left(B_{1}\right)} \leq C \varepsilon_{0}
$$

Then we have

$$
\int_{B_{1}}\left|u_{t}\left(t_{2}\right)\right|^{2} \leq \int_{B_{1}}\left|u_{t}\left(t_{1}\right)\right|^{2}
$$

In particular, Proposition 2.1 holds if (2-12) and (2-13) are valid for any $t_{0} \in\left[t_{1}, t_{2}\right]$ and any $t_{2}>t_{1} \geq$ $T_{0} \geq 1$.

Proof. Differentiating the flow equation (1-8) with respect to $t$, multiplying with $u_{t}$, and integrating over $B_{1} \times\left[t_{1}, t_{2}\right]$, we have

$$
\begin{aligned}
\frac{1}{2} \int_{t_{1}}^{t_{2}} \int_{B_{1}} \partial_{t}\left|u_{t}\right|^{2}+\int_{t_{1}}^{t_{2}} \int_{B_{1}}\left|\nabla u_{t}\right|^{2} & \leq C \int_{t_{1}}^{t_{2}} \int_{B_{1}}\left|u_{t}\right|^{2}|\nabla u|^{2}+\left|u_{t}\right||\nabla u|\left|\nabla u_{t}\right| \\
& \leq \frac{1}{2} \int_{t_{1}}^{t_{2}} \int_{B_{1}}\left|\nabla u_{t}\right|^{2}+C \int_{t_{1}}^{t_{2}} \int_{B_{1}}\left|u_{t}\right|^{2}|\nabla u|^{2}
\end{aligned}
$$

Since (2-12) and (2-13) are valid for any $t_{0} \in\left[t_{1}, t_{2}\right]$, we can use the same arguments as in the proof of Lemma 2.4 to get an estimate for $\int_{B_{1}}\left|u_{t}\right|^{2}|\nabla u|^{2}$ at the time $t_{0}$ slice. Indeed, similarly to (2-5) (that is, 
replacing $u\left(t_{1}\right)-u\left(t_{2}\right)$ by $\left.u_{t}\left(t_{0}\right)\right)$, for any $t_{0} \in\left[t_{1}, t_{2}\right]$, we have

$$
\int_{B_{1}}\left|u_{t}\right|^{2}|\nabla u|^{2}\left(t_{0}\right) \leq C\|\psi\|_{L^{\infty}} \int_{B_{1}}\left|\nabla u_{t}\left(t_{0}\right)\right|^{2} \leq C \varepsilon_{0} \int_{B_{1}}\left|\nabla u_{t}\left(t_{0}\right)\right|^{2} .
$$

Inserting (2-16) back into (2-15) (for any $t_{0} \in\left[t_{1}, t_{2}\right]$ ), we see that the right-hand side of (2-15) can be absorbed into the left-hand side if we choose $\varepsilon_{0}$ sufficiently small. This implies that we have (2-14) for any such $t_{2}>t_{1} \geq T_{0}$. In the above calculations, we should treat $u_{t}$ as a difference quotient: $u_{t}(\cdot, t)=\lim _{h \rightarrow 0^{+}}(u(\cdot, t+h)-u(\cdot, t)) / h$, which is zero on $\partial B_{1}$ for all $t \geq 1$; moreover, we have denoted $\nabla u_{t}(\cdot, t)=\lim _{h \rightarrow 0^{+}}(\nabla(u(\cdot, t+h)-u(\cdot, t))) / h$ and all the calculations are valid for any fixed $h>0$. We then we take $h \rightarrow 0^{+}$to conclude (2-14).

If (2-12) and (2-13) are valid for any $t_{0} \in\left[t_{1}, t_{2}\right]$ and any $t_{2}>t_{1} \geq T_{0} \geq 1$, then, in view of (2-14), estimating by the mean value of $\left|u_{t}\right|^{2}$ over $B_{1} \times\left[t_{1}, t_{2}\right]$ gives Proposition 2.1.

Therefore, everything boils down to validating the assumptions in Lemmas 2.4 and 2.5, that is, the existence of such functions $\psi$ satisfying (2-7), (2-8) and (2-12), (2-13), respectively, for any $t_{0} \geq T_{0}$ for some $T_{0} \geq 1$. We point out that, a priori we only know that the energy density $|\nabla u(t)|^{2} \operatorname{lies}$ in $L^{1}\left(B_{1}\right)$ with global estimate $\left\||\nabla u(t)|^{2}\right\|_{L^{1}\left(B_{1}\right)} \leq \varepsilon_{0}$ for any fixed $t$. But $L^{1}$ is the borderline case in which the standard $L^{p}$-theory for the Dirichlet problem (2-7) with estimate (2-8) fails!

However, the following regularity theorem for boundary value problems in the local Hardy space $h^{1}\left(B_{1}\right)$ sheds new light on the problem of validating the assumptions in Lemmas 2.4 and 2.5. Here the local Hardy space $h^{1}\left(B_{1}\right)$ is a strict subspace of $L^{1}\left(B_{1}\right)$ and we will recall its definition in Definition 2.8 below.

Theorem 2.6 (cf. [Semmes 1994, Theorem 1.100; Chang et al. 1993, Theorem 5.1]). Let $f \in h^{1}\left(B_{1}\right)$ such that $f \geq 0$ a.e. in $B_{1}$. Then there exists a function $\psi \in L^{\infty} \cap W_{0}^{1,2}\left(B_{1}\right)$ solving the Dirichlet problem

$$
\begin{cases}\Delta \psi=f & \text { in } B_{1} \\ \psi=0 & \text { on } \partial B_{1}\end{cases}
$$

Moreover, there exists a constant $C>0$ such that

$$
\|\psi\|_{L^{\infty}\left(B_{1}\right)}+\|\nabla \psi\|_{L^{2}\left(B_{1}\right)} \leq C\|f\|_{h^{1}\left(B_{1}\right)} .
$$

Proof. For self-containedness, we include an elementary proof of this theorem in Appendix A.

Remark 2.7. This theorem can be thought of as a generalization of a result from [Müller 1990]; cf. Wente's lemma (Lemma 3.6). For a more general version of this theorem, we refer to Chang, Krantz, and Stein's work [Chang et al. 1993].

Definition 2.8 [Miyachi 1990]. Choose a Schwartz function $\phi \in C_{0}^{\infty}\left(B_{1}\right)$ such that $\int_{B_{1}} \phi d x=1$ and let $\phi_{t}(x)=t^{-2} \phi(x / t)$. For a measurable function $f$ defined in $B_{1}$, we say that $f$ lies in the local Hardy space $h^{1}\left(B_{1}\right)$ if the radial maximal function of $f$

$$
f^{*}(x)=\sup _{0<t<1-|x|}\left|\int_{B_{t}(x)} \frac{1}{t^{2}} \phi\left(\frac{x-y}{t}\right) f(y) d y\right|(x)=\sup _{0<t<1-|x|}\left|\phi_{t} * f\right|(x)
$$


belongs to $L^{1}\left(B_{1}\right)$ and we define

$$
\|f\|_{h^{1}\left(B_{1}\right)}=\left\|f^{*}(x)\right\|_{L^{1}\left(B_{1}\right)} .
$$

It follows immediately that $h^{1}\left(B_{1}\right)$ is a strict subspace of $L^{1}\left(B_{1}\right)$ and

$$
\|f\|_{L^{1}\left(B_{1}\right)} \leq\|f\|_{h^{1}\left(B_{1}\right)} .
$$

It is also clear that if $f \in L^{p}\left(B_{1}\right)$ for some $p>1$, then $\|f\|_{h^{1}\left(B_{1}\right)} \leq C\|f\|_{L^{p}\left(B_{1}\right)}$.

We remark that the local Hardy spaces $h^{1}$ (or the global version $\mathscr{H}^{1}$ ) act as replacements for $L^{1}$ in Calderon-Zygmund estimates. Therefore, by Theorem 2.6, if we can somehow manage to obtain a "slightly" improved global estimate for $|\nabla u|^{2}$ from $L^{1}\left(B_{1}\right)$ to $h^{1}\left(B_{1}\right)$ for all $t_{0} \geq T_{0}$, it will be sufficient to validate the assumptions in Lemmas 2.4 and 2.5. As mentioned above, the subtlety is that, without imposing any regularity information on the boundary data $\chi$, global estimates are very difficult to obtain.

The rest of the paper is devoted to validating the assumptions in Lemmas 2.4 and 2.5. Namely, in view of Theorem 2.6, it suffices to show there exists $T_{0}>0$ such that

$$
\left\|\left|\nabla u\left(t_{0}\right)\right|^{2}\right\|_{h^{1}\left(B_{1}\right)} \leq C \varepsilon_{0} \quad \text { for any } t_{0} \geq T_{0} .
$$

The point here is that no pointwise estimate on $\nabla u$ such as (2-6) is needed, and instead, a (weaker) improved global integral estimate (2-21) will be sufficient and turns out to be the key to the proof of Theorem 1.6.

\section{Analysis of harmonic map heat flow using Rivière's gauge}

Regarding the regularity of weakly harmonic maps from surfaces, Hélein (see, for example, [Hélein 2002]) proved the interior regularity with the help of the so-called Coulomb or moving frame, and Qing [1995] showed the continuity up to the boundary in the case of continuous boundary data based on Hélein's technique. Rivière [2007] succeeded in writing the 2-dimensional conformally invariant nonlinear system of elliptic PDE's (which includes the weakly harmonic map equation (1-2)) in the form

$$
-\Delta u^{i}=\Omega_{j}^{i} \cdot \nabla u^{j}, \quad i=1,2, \ldots, n, \quad \text { or } \quad-\Delta u=\Omega \cdot \nabla u
$$

with $\Omega=\left(\Omega_{j}^{i}\right)_{1 \leq i, j \leq n} \in L^{2}\left(B_{1}, \mathfrak{s o}(n) \otimes \bigwedge^{1} \mathbb{R}^{2}\right)$ and $\Omega_{j}^{i}=-\Omega_{i}^{j}$ (antisymmetry). Here and throughout the paper, the Einstein summation convention is used. We refer to the system of equations (3-1) as Rivière's equation. This special form of the nonlinearity enabled Rivière to obtain a conservation law for this system of PDE's (see (3-8) below), which is accomplished via a technique that we call Rivière's gauge decomposition. More precisely, following the strategy of [Uhlenbeck 1982], Rivière [2007] used an algebraic feature of $\Omega$ - its antisymmetry - to construct $\xi \in W_{0}^{1,2}\left(B_{1}, \mathfrak{s o}(n)\right)$ and a gauge transformation matrix $P \in W^{1,2} \cap L^{\infty}\left(B_{1}, \mathrm{SO}(n)\right)$ (which pointwise almost everywhere is an orthogonal matrix in $\mathbb{R}^{n \times n}$ ) satisfying some good properties. 
Theorem 3.1 [Rivière 2007, Lemma A.3]. There exist $\varepsilon>0$ and $C>0$ such that, for every $\Omega$ in $L^{2}\left(B_{1}, \mathfrak{s o}(n) \otimes \bigwedge^{1} \mathbb{R}^{2}\right)$ satisfying

$$
\int_{B_{1}}|\Omega|^{2} \leq \varepsilon
$$

there exist $\xi \in W_{0}^{1,2}\left(B_{1}, \mathfrak{s o}(n)\right)$ and $P \in W^{1,2}\left(B_{1}, \mathrm{SO}(n)\right)$ such that

$$
\nabla^{\perp} \xi=P^{T} \nabla P+P^{T} \Omega P \text { in } B_{1} \quad \text { with } \xi=0 \text { on } \partial B_{1},
$$

and

$$
\|\nabla \xi\|_{L^{2}\left(B_{1}\right)}+\|\nabla P\|_{L^{2}\left(B_{1}\right)} \leq C\|\Omega\|_{L^{2}\left(B_{1}\right)} .
$$

Here the superscript $T$ denotes the transpose of a matrix.

Remark 3.2. Multiplying both sides of (3-2) by $P$ from the left gives (with indices and $1 \leq m, z \leq n$ )

$$
\nabla P_{j}^{i}=P_{m}^{i} \nabla^{\perp} \xi_{j}^{m}-\Omega_{z}^{i} P_{j}^{z}, \quad 1 \leq i, j \leq n .
$$

Remark 3.3. Besides Uhlenbeck's method there is another way to construct the gauge transformation matrix $P$, namely, one can minimize the energy functional

$$
E(R)=\int_{B_{1}}\left|R^{T} \nabla R+R^{T} \Omega R\right|^{2}
$$

among all $R \in W^{1,2}\left(B_{1}, \mathrm{SO}(n)\right)$; see, for example, [Choné 1995; Schikorra 2010].

Another key result from Rivière's work is the following theorem, which was proved based on Theorem 3.1.

Theorem 3.4 [Rivière 2007, Theorem I.4]. There exist $\varepsilon>0$ and $C>0$ such that, for every $\Omega$ in $L^{2}\left(B_{1}, \mathfrak{s o}(n) \otimes \bigwedge^{1} \mathbb{R}^{2}\right)$ satisfying

$$
\int_{B_{1}}|\Omega|^{2} \leq \varepsilon
$$

there exist

$$
\widehat{A} \in W^{1,2} \cap C^{0}\left(B_{1}, G l_{n}(\mathbb{R})\right), \quad A=(\widehat{A}+\mathrm{Id}) P^{T} \in L^{\infty} \cap W^{1,2}\left(B_{1}, G l_{n}(\mathbb{R})\right), \quad B \in W_{0}^{1,2}\left(B_{1}, M_{n}(\mathbb{R})\right)
$$

such that

$$
\nabla A-A \Omega=\nabla^{\perp} B
$$

and

$$
\|\widehat{A}\|_{W^{1,2}\left(B_{1}\right)}+\|\widehat{A}\|_{L^{\infty}\left(B_{1}\right)}+\|B\|_{W^{1,2}\left(B_{1}\right)} \leq C\|\Omega\|_{L^{2}\left(B_{1}\right)} .
$$

Remark 3.5. Combining (3-6) with (3-1), one obtains the conservation law (in the distribution sense) for Rivière's equation, (3-1):

$$
\operatorname{div}\left(A \nabla u+B \nabla^{\perp} u\right)=0
$$


Equation (3-1), first considered in such generality in [Rivière 2007], generalizes a number of interesting equations appearing naturally in geometry, including the harmonic map equation (1-2), the $H$-surface equation, and, more generally, the Euler-Lagrange equation of any conformally invariant elliptic Lagrangian which is quadratic in the gradient. We remark that the harmonic map equation (1-2) can be written in the form of (3-1) if we set

$$
\Omega:=\left(\Omega_{j}^{i}\right)_{1 \leq i, j \leq n}, \quad \text { where } \Omega_{j}^{i}:=\left[\Pi^{i}(u)_{j, l}-\Pi^{j}(u)_{i, l}\right] \nabla u^{l} .
$$

A central issue is the regularity of the weak solution $u$ to this system of equations (3-1). Based on the conservation law (3-8), Rivière proved the (interior) continuity of any $W^{1,2}$ weak solution $u$ to (3-1). This also resolved two conjectures by Heinz and Hildebrandt, respectively; see [Rivière 2007]. We point out that the harmonic map heat flow (1-8) on $B_{1}$ can be written in the form

$$
u_{t}-\Delta u=\Omega \cdot \nabla u \quad \text { on } B_{1} \times(0, T),
$$

where $\Omega$ is as in (3-9).

The deep reason for Rivière's argument to work is that once the conservation law (3-8) is established, (3-1) can be rewritten in the form

$$
\operatorname{div}(A \nabla u)=\nabla^{\perp} B \cdot \nabla u .
$$

The right-hand side of this new equation lies in the Hardy space $\mathscr{H}^{1}$ by a result of Coifman, Lions, Meyer, and Semmes [Coifman et al. 1993]. Moreover, using a Hodge decomposition argument, one can show that $u$ lies locally in $W^{2,1}$, which embeds into $C^{0}$ in two dimensions; cf. the proof of Theorem 3.7 below. The key to this fact is a special "compensation phenomena" for Jacobian determinants, first observed in [Wente 1969]. We will refer to the following lemma of Wente, for which an elementary proof can be found in [Brezis and Coron 1983; Hélein 2002, Theorem 3.1.2], and which will be the key ingredient of our proof.

Lemma 3.6 [Wente 1969]. If $a, b \in W^{1,2}\left(B_{1}, \mathbb{R}\right)$ and $w$ is the solution of

$$
\left\{\begin{array}{cc}
\Delta w=\frac{\partial a}{\partial y} \frac{\partial b}{\partial x}-\frac{\partial a}{\partial x} \frac{\partial b}{\partial y}=\nabla a \cdot \nabla^{\perp} b & \text { in } B_{1}, \\
w=0 \text { or } \frac{\partial w}{\partial v}=0 & \text { on } \partial B_{1},
\end{array}\right.
$$

then $w \in C^{0} \cap W^{1,2}\left(B_{1}, \mathbb{R}\right)$ and the estimate

$$
\|w\|_{L^{\infty}\left(B_{1}\right)}+\|\nabla w\|_{L^{2}\left(B_{1}\right)} \leq C\|\nabla a\|_{L^{2}\left(B_{1}\right)}\|\nabla b\|_{L^{2}\left(B_{1}\right)}
$$

holds, where we choose $\int_{B_{1}} w=0$ for the Neumann boundary data.

Now let $u(x, t) \in W^{1,2}\left(B_{1} \times[0, \infty), \mathcal{N}\right)$ be a global weak solution to the harmonic map heat flow (1-8) with $E\left(u_{0}\right)<\varepsilon_{0}, E(u(\cdot, t))$ nonincreasing in $t$, and $\left.u(\cdot, t)\right|_{\partial B_{1}}=\chi$ for all $t \geq 0$ as in Theorem 1.6. First note that, for a.e. $t_{0} \in(0, \infty)$, we have $u_{t}\left(t_{0}\right) \in L^{2}\left(B_{1}\right)$. Then, for any fixed $t_{0}$ such that $u_{t}\left(t_{0}\right) \in L^{2}\left(B_{1}\right)$, as in (3-9), we have

$$
\Omega\left(t_{0}\right)=\left(\Omega_{j}^{i}\left(t_{0}\right)\right)_{1 \leq i, j \leq n}, \quad \text { where } \Omega_{j}^{i}\left(t_{0}\right)=\left[\Pi^{i}\left(u\left(t_{0}\right)\right)_{j, l}-\Pi^{j}\left(u\left(t_{0}\right)\right)_{i, l}\right] \nabla u^{l}\left(t_{0}\right) .
$$


We will express this by writing $\Omega\left(t_{0}\right)=\Pi\left(u\left(t_{0}\right)\right) \nabla u\left(t_{0}\right)$. Moreover,

$$
\int_{B_{1}}\left|\Omega\left(t_{0}\right)\right|^{2} \leq C E\left(u\left(t_{0}\right)\right) \leq C \varepsilon_{0} .
$$

Therefore Rivière's theorems on the existence of gauge (Theorems 3.1 and 3.4) apply to this time $t_{0}$ slice, and we find the existence of matrices $P\left(t_{0}\right) \in W^{1,2}\left(B_{1}, \mathrm{SO}(n)\right)$,

$$
A\left(t_{0}\right)=\left(\widehat{A}\left(t_{0}\right)+\mathrm{Id}\right) P^{T}\left(t_{0}\right) \in L^{\infty} \cap W^{1,2}\left(B_{1}, G l_{n}(\mathbb{R})\right),
$$

and

$$
B\left(t_{0}\right) \in W_{0}^{1,2}\left(B_{1}, M_{n}(\mathbb{R})\right)
$$

such that

$$
\nabla A\left(t_{0}\right)-A\left(t_{0}\right) \Omega\left(t_{0}\right)=\nabla^{\perp} B\left(t_{0}\right)
$$

with the corresponding estimates (3-3) and (3-7).

Combining (3-14) with the harmonic map heat flow equation (3-10) yields (omitting the index $t_{0}$ )

$$
\begin{aligned}
\operatorname{div}\left(A \nabla u+B \nabla^{\perp} u\right) & =\nabla A \cdot \nabla u+A \Delta u+\nabla B \cdot \nabla^{\perp} u \\
& =\nabla A \cdot \nabla u+A\left(-\Omega \cdot \nabla u+u_{t}\right)+\nabla B \cdot \nabla^{\perp} u \\
& =\nabla A \cdot \nabla u+\left(\nabla^{\perp} B-\nabla A\right) \cdot \nabla u+A u_{t}+\nabla B \cdot \nabla^{\perp} u \\
& =A u_{t} .
\end{aligned}
$$

We refer to (3-15) as an almost conservation law. By the results of [Coifman et al. 1993] and the standard $L^{p}$ theory, (3-15) readily implies that $u\left(t_{0}\right) \in C^{0}\left(B_{1}, \mathbb{R}^{n}\right)$. In fact, we have the following $\varepsilon$-regularity theorem.

Theorem 3.7. There exist $\varepsilon_{0}>0$ depending only on $\mathcal{N}$ such that if $u \in W^{1,2}\left(B_{1} \times[0, \infty), \mathcal{N}\right)$ is a global weak solution to the harmonic map heat flow (1-8) with $E\left(u_{0}\right)<\varepsilon_{0}, E(u(\cdot, t))$ nonincreasing in $t$, and $\left.u(\cdot, t)\right|_{\partial B_{1}}=\chi$ for all $t \geq 0$, then $u \in C^{\infty}\left(B_{1} \times[1, \infty), \mathcal{N}\right)$.

Proof. For any fixed $t_{0}$ such that $u_{t}\left(t_{0}\right) \in L^{2}\left(B_{1}\right)$, by Hodge decomposition (see, for example, [Iwaniec and Martin 2001, Corollary 10.5.1]), there exist $D\left(t_{0}\right), E\left(t_{0}\right) \in W^{1,2}\left(B_{1}, \mathbb{R}^{n}\right)$ such that (omitting the index $t_{0}$ )

$$
A \nabla u=\nabla D+\nabla^{\perp} E
$$

Note that (3-15) implies

$$
\left\{\begin{array}{l}
\operatorname{div}(A \nabla u)=-\nabla B \cdot \nabla^{\perp} u+A u_{t}, \\
\operatorname{curl}(A \nabla u)=\nabla^{\perp} A \cdot \nabla u
\end{array}\right.
$$

Combining (3-16) and (3-17), we have

$$
\left\{\begin{array}{l}
\Delta D=-\nabla B \cdot \nabla^{\perp} u+A u_{t}, \\
\Delta E=\nabla^{\perp} A \cdot \nabla u
\end{array}\right.
$$

Then, by the results of [Coifman et al. 1993] and via an extension argument, using the fact that $A u_{t}\left(t_{0}\right) \in L^{2}\left(B_{1}\right)$, we get $A \nabla u\left(t_{0}\right) \in W_{\text {loc }}^{1,1}\left(B_{1}\right)$. Therefore $u\left(t_{0}\right) \in W_{\text {loc }}^{2,1}\left(B_{1}\right)$, which embeds into $C^{0}\left(B_{1}\right)$. 
Indeed, $A \nabla u\left(t_{0}\right) \in W_{\text {loc }}^{1,1}\left(B_{1}\right)$ implies immediately that

$$
\Omega\left(t_{0}\right)=\Pi\left(u\left(t_{0}\right)\right) \nabla u\left(t_{0}\right) \in W_{\mathrm{loc}}^{1,1}\left(B_{1}\right) .
$$

Then, by [Rivière 2012, Theorem IV.4], the flow equation (3-10), (3-19), and the fact that $u_{t}\left(t_{0}\right) \in L^{2}\left(B_{1}\right)$ yield that $\nabla u\left(t_{0}\right) \in L_{\mathrm{loc}}^{p}\left(B_{1}\right)$ for some $p>2$. Note that this is valid for a.e. $t_{0} \in(0, \infty)$. Then, via a standard bootstrapping argument, we have $\nabla u \in L_{\text {loc }}^{q}\left(B_{1} \times[1, T]\right.$ ) for all $q>1$ and any $T>1$ (see, for example, [Lieberman 1996]) and all higher order interior regularity follows.

Again, we see that the "compensation phenomenon" enjoyed by the special Jacobian structure (see Lemma 3.6) has played an important role here, and these Wente-type estimates have many interesting applications, as in [Wente 1969; Brezis and Coron 1983; 1984; Tartar 1985; Coifman et al. 1993; Hélein 2002; Rivière 2007; 2008; 2011; Lamm and Lin 2013].

\section{Improved estimates on the matrices $B$ and $P$}

Our main observation in this section is the existence of hidden Jacobian structures for $\Delta B$ and $\Delta P$, valid only when $\Omega$ is of some special form: in our case, $\Omega=\Pi(u) \nabla u$. This will allow us to gain an improved global estimate for the matrix $B$ and an improved local estimate for $P$. We start with the improved estimate for $B$.

Proposition 4.1. Let $u(x, t)$ be as in Theorem 1.6. For any $t_{0} \in[1, \infty)$, we have

$$
\left\|B\left(t_{0}\right)\right\|_{L^{\infty}\left(B_{1}\right)} \leq C \int_{B_{1}}\left|\nabla u\left(t_{0}\right)\right|^{2} \leq C \varepsilon_{0} .
$$

Proof. We recall that $\Omega$ is given by $\Pi(u) \nabla u$ as in (3-9) and therefore $\left\|\Omega\left(t_{0}\right)\right\|_{L^{2}\left(B_{1}\right)}^{2} \leq C \varepsilon_{0}$ for all $t_{0} \geq 1$. Now let $\varepsilon_{0}$ be so small that Theorems 3.1 and 3.4 apply. Taking the curl on both sides of (3-14) yields

$$
\Delta B\left(t_{0}\right)=-\operatorname{curl}\left(A\left(t_{0}\right) \Pi\left(u\left(t_{0}\right)\right) \nabla u\left(t_{0}\right)\right)=-\nabla u\left(t_{0}\right) \cdot \nabla^{\perp}\left(A\left(t_{0}\right) \Pi\left(u\left(t_{0}\right)\right)\right) .
$$

Combining the Jacobian structure of the right-hand side of (4-2) with the zero boundary condition of $B$ and estimates (3-3) and (3-7), Lemma 3.6 gives (4-1). Here we have also used $E(u(t))<\varepsilon_{0}$ for all $t \geq 0$ and the smoothness and compactness of the target manifold $\mathcal{N}$.

Next, as a step toward the improved local estimate on the matrix $P$, we show that $\Delta P$ also has a special Jacobian structure.

Lemma 4.2. Let $u(x, t)$ be as in Theorem 1.6. For any $t_{0} \in[1, \infty)$ such that $u_{t}\left(\cdot, t_{0}\right) \in L^{2}\left(B_{1}\right)$, there exist

$$
\xi\left(t_{0}\right) \in W_{0}^{1,2}\left(B_{1}, \mathfrak{s o}(n)\right), \quad \eta\left(t_{0}\right) \in W^{1,2}\left(B_{1}, \mathbb{R}^{n}\right), \quad \zeta\left(t_{0}\right) \in W_{0}^{2,2}\left(B_{1}, \mathbb{R}^{n}\right)
$$

and

$$
Q_{k}\left(t_{0}\right), R_{k}\left(t_{0}\right) \in W^{1,2} \cap L^{\infty}\left(B_{1}, G l_{n}(\mathbb{R})\right), \quad k=1, \ldots, n
$$

with

$\left\|\nabla \xi\left(t_{0}\right)\right\|_{L^{2}\left(B_{1}\right)}+\left\|\nabla \eta\left(t_{0}\right)\right\|_{L^{2}\left(B_{1}\right)}+\left\|\nabla \zeta\left(t_{0}\right)\right\|_{L^{2}\left(B_{1}\right)}+\sum_{k}\left(\left\|\nabla Q_{k}\left(t_{0}\right)\right\|_{L^{2}\left(B_{1}\right)}+\left\|\nabla R_{k}\left(t_{0}\right)\right\|_{L^{2}\left(B_{1}\right)}\right) \leq C \sqrt{\varepsilon_{0}}$ 
and

$$
\left\|\zeta\left(t_{0}\right)\right\|_{W^{2,2}\left(B_{1}\right)} \leq C\left\|u_{t}\left(t_{0}\right)\right\|_{L^{2}\left(B_{1}\right)}
$$

such that

$$
\Delta P\left(t_{0}\right)=\nabla P\left(t_{0}\right) \cdot \nabla^{\perp} \xi\left(t_{0}\right)+\nabla Q_{k}\left(t_{0}\right) \cdot \nabla^{\perp} \eta^{k}\left(t_{0}\right)+\nabla R_{k}\left(t_{0}\right) \cdot \nabla^{\perp} u^{k}\left(t_{0}\right)+\operatorname{div}\left(Q_{k}\left(t_{0}\right) \nabla \zeta^{k}\left(t_{0}\right)\right) .
$$

Proof. We omit the index $t_{0}$ in the proof. Hodge decomposition and the estimates for the $L^{\infty}$-norms of $A$ and $B$ imply the existence of $\eta \in W^{1,2}\left(B_{1}, \mathbb{R}^{n}\right)$ and $\zeta \in W_{0}^{1,2}\left(B_{1}, \mathbb{R}^{n}\right)$ such that

$$
\nabla^{\perp} \eta+\nabla \zeta=A \nabla u+B \nabla^{\perp} u
$$

with

$$
\|\nabla \eta\|_{L^{2}\left(B_{1}\right)}+\|\nabla \zeta\|_{L^{2}\left(B_{1}\right)} \leq C\|\nabla u\|_{L^{2}\left(B_{1}\right)} \leq C \sqrt{\varepsilon_{0}} .
$$

Moreover, by the almost conservation law (3-15), we have

$$
\Delta \zeta=A u_{t} \in L^{2}\left(B_{1}\right) \quad \text { and }\left.\quad \zeta\right|_{\partial B_{1}}=0,
$$

which gives (4-3) by the standard $L^{p}$-theory. Multiplying both sides of (4-5) by $A^{-1}$ from the left gives (with indices)

$$
\nabla u^{l}=\left(A^{-1}\right)_{k}^{l} \nabla^{\perp} \eta^{k}-\left(A^{-1} B\right)_{k}^{l} \nabla^{\perp} u^{k}+\left(A^{-1}\right)_{k}^{l} \nabla \zeta^{k}, \quad l=1,2, \ldots, n .
$$

Taking the divergence on both sides of (3-4) yields

$$
\Delta P_{j}^{i}=\nabla P_{m}^{i} \cdot \nabla^{\perp} \xi_{j}^{m}-\operatorname{div}\left(\Omega_{z}^{i} P_{j}^{z}\right), \quad 1 \leq i, j \leq n .
$$

Since $\Omega_{z}^{i}=\left[\Pi^{i}(u)_{z, l}-\Pi^{z}(u)_{i, l}\right] \nabla u^{l}$, combining (4-7) and (4-8) gives

$$
\begin{aligned}
& \Delta P_{j}^{i} \\
& =\nabla P_{m}^{i} \cdot \nabla^{\perp} \xi_{j}^{m}-\operatorname{div}\left[\left(\Pi^{i}(u)_{z, l}-\Pi^{z}(u)_{i, l}\right)\left(\left(A^{-1}\right)_{k}^{l} \nabla^{\perp} \eta^{k}-\left(A^{-1} B\right)_{k}^{l} \nabla^{\perp} u^{k}+\left(A^{-1}\right)_{k}^{l} \nabla \zeta^{k}\right) P_{j}^{z}\right] \\
& =\nabla P_{m}^{i} \cdot \nabla^{\perp} \xi_{j}^{m}-\nabla\left[\left(\Pi^{i}(u)_{z, l}-\Pi^{z}(u)_{i, l}\right) P_{j}^{z}\left(A^{-1}\right)_{k}^{l}\right] \cdot \nabla^{\perp} \eta^{k} \\
& \quad+\nabla\left[\left(\Pi^{i}(u)_{z, l}-\Pi^{z}(u)_{i, l}\right) P_{j}^{z}\left(A^{-1} B\right)_{k}^{l}\right] \cdot \nabla^{\perp} u^{k}-\operatorname{div}\left[\left(\left(\Pi^{i}(u)_{z, l}-\Pi^{z}(u)_{i, l}\right) P_{j}^{z}\left(A^{-1}\right)_{k}^{l}\right) \nabla \zeta^{k}\right] .
\end{aligned}
$$

Defining

and

$$
\left(Q_{k}\right)_{j}^{i}=-\left(\Pi^{i}(u)_{z, l}-\Pi^{z}(u)_{i, l}\right) P_{j}^{z}\left(A^{-1}\right)_{k}^{l}
$$

$$
\left(R_{k}\right)_{j}^{i}=\left(\Pi^{i}(u)_{z, l}-\Pi^{z}(u)_{i, l}\right) P_{j}^{z}\left(A^{-1} B\right)_{k}^{l},
$$

where $1 \leq k, i, j \leq n$, completes the proof.

Next we prove a local estimate on the oscillation of the matrix $P$ based on Lemma 4.2. A key observation here is that whether a function is in the local Hardy space $h^{1}\left(B_{1}\right)$ essentially depends on its local behavior (see Definition 2.8). This local oscillation estimate on $P$ provides important information that we need to control the local behavior of $|\nabla u|^{2}$. This point will become apparent in Section 5. As we shall see, the Jacobian structure of $\Delta P$ enters in a crucial way. 
Lemma 4.3. Let $u(x, t)$ be as in Theorem 1.6. For any $t_{0} \in[1, \infty)$ such that $u_{t}\left(\cdot, t_{0}\right) \in L^{2}\left(B_{1}\right)$, any $x \in B_{1}$, any $r>0$ such that $B_{2 r}(x) \subset B_{1}$, and any $y \in B_{r}(x)$, we have

$$
\left|P\left(y, t_{0}\right)-P\left(x, t_{0}\right)\right| \leq C\left(\sqrt{\varepsilon_{0}}+\left\|u_{t}\left(t_{0}\right)\right\|_{L^{2}\left(B_{1}\right)}\right) .
$$

Proof. We will omit the index $t_{0}$ in the proof. Let $\widetilde{P} \in W^{1,2}\left(B_{1}, M_{n}(\mathbb{R})\right)$ be the weak solution of

$$
\begin{cases}\Delta \widetilde{P}=\nabla P \cdot \nabla^{\perp} \xi+\nabla Q_{k} \cdot \nabla^{\perp} \eta^{k}+\nabla R_{k} \cdot \nabla^{\perp} u^{k}+\operatorname{div}\left(Q_{k} \nabla \zeta^{k}\right) & \text { in } B_{1}, \\ \widetilde{P}=0 & \text { on } \partial B_{1},\end{cases}
$$

where $Q_{k}, R_{k}, \eta^{k}$, and $\zeta^{k}$ are from Lemma 4.2.

Then, by Wente's lemma (Lemma 3.6) and the standard $L^{p}$-theory (and $W^{2,2}\left(B_{1}\right) \hookrightarrow C^{0}\left(B_{1}\right)$ ), we have $\widetilde{P} \in C^{0}\left(B_{1}, M_{n}(\mathbb{R})\right)$ and

$$
\|\widetilde{P}\|_{L^{\infty}\left(B_{1}\right)}+\|\nabla \widetilde{P}\|_{L^{2}\left(B_{1}\right)} \leq C\left(\varepsilon_{0}+\left\|u_{t}\left(t_{0}\right)\right\|_{L^{2}\left(B_{1}\right)}\right) .
$$

Since

$$
\Delta(P-\widetilde{P})=0 \quad \text { in } B_{1},
$$

we know that $V=P-\widetilde{P} \in C^{\infty}\left(B_{1}, M_{n}(\mathbb{R})\right)$ is harmonic. Now, for any $x \in B_{1}$, any $r>0$ such that $B_{2 r}(x) \subset B_{1}$, and any $y \in B_{r}(x)$, we have

$$
\begin{aligned}
|V(y)-V(x)| & \leq C r\|\nabla V\|_{L^{\infty}\left(B_{r}(x)\right)} \leq C r^{-1}\|\nabla V\|_{L^{1}\left(B_{2 r}(x)\right)} \\
& \leq C\|\nabla V\|_{L^{2}\left(B_{2 r}(x)\right)} \leq C\left(\|\nabla P\|_{L^{2}\left(B_{2 r}(x)\right)}+\|\nabla \widetilde{P}\|_{L^{2}\left(B_{2 r}(x)\right)}\right) \\
& \leq C\left(\sqrt{\varepsilon_{0}}+\left\|u_{t}\left(t_{0}\right)\right\|_{L^{2}\left(B_{1}\right)}\right)
\end{aligned}
$$

where we have used the mean value property of $V$ and (4-11), (3-3). Combining (4-11) and (4-12) yields that, for any $x \in B_{1}$, any $r>0$ such that $B_{2 r}(x) \subset B_{1}$, and any $y \in B_{r}(x)$, we have

$$
\left|P\left(y, t_{0}\right)-P\left(x, t_{0}\right)\right| \leq C\left(\sqrt{\varepsilon_{0}}+\left\|u_{t}\left(t_{0}\right)\right\|_{L^{2}\left(B_{1}\right)}\right)
$$

which gives the desired estimate (4-10).

\section{Validation of (2-21) and completion of the proof of Theorem 1.6}

With the results so far at our disposal, we are now in a position to validate (2-21). As mention above, the local estimate on the oscillation of the transformation matrix $P$ in Lemma 4.3 will be the key ingredient.

Lemma 5.1. Let $u(x, t)$ be as in Theorem 1.6. For any $t_{0} \in[1, \infty)$ such that $\left\|u_{t}\left(t_{0}\right)\right\|_{L^{2}\left(B_{1}\right)}<\sqrt{\varepsilon_{0}}$, we have

$$
\left|\nabla u\left(t_{0}\right)\right|^{2} \in h^{1}\left(B_{1}\right)
$$

with the estimate

$$
\left\|\left|\nabla u\left(t_{0}\right)\right|^{2}\right\|_{h^{1}\left(B_{1}\right)} \leq C \varepsilon_{0} .
$$


Remark 5.2. Lemma 5.1 continues to hold for the flow (3-10) with a more general $\Omega$ in the form $\Omega_{j}^{i}=\sum_{l=1}^{n} f_{j l}^{i} \nabla u^{l}+g_{j l}^{i} \nabla^{\perp} u^{l}$ (which includes $\Omega=\Omega_{j}^{i}=\left[\Pi^{i}(u)_{j, l}-\Pi^{j}(u)_{i, l}\right] \nabla u^{l}$ for the harmonic map heat flow as a special case); see [Lamm and Lin 2013]. Moreover, the condition $\left\|u_{t}\left(t_{0}\right)\right\|_{L^{2}\left(B_{1}\right)}<\varepsilon_{0}$ can be replaced by the fact that $\left\|u_{t}\left(t_{0}\right)\right\|_{L^{p}\left(B_{1}\right)}$ is sufficiently small for some $p>1$.

Proof of Lemma 5.1. By the assumption $\left\|u_{t}\left(t_{0}\right)\right\|_{L^{2}\left(B_{1}\right)}<\sqrt{\varepsilon_{0}}$ and Lemma 4.3, for any $x \in B_{1}$, any $r>0$ such that $B_{2 r}(x) \subset B_{1}$, and any $y \in B_{r}(x)$, we have

$$
\left|P\left(y, t_{0}\right)-P\left(x, t_{0}\right)\right| \leq C \sqrt{\varepsilon_{0}} .
$$

We will omit the index $t_{0}$ from now on. By Proposition 4.1 and Theorems 3.1 and 3.4, for any $x \in B_{1}$, any $r>0$ such that $B_{2 r}(x) \subset B_{1}$, and any $y \in B_{r}(x)$, we have (choosing $\varepsilon_{0}$ sufficiently small)

$$
\begin{aligned}
0 & \leq \frac{1}{2}|\nabla u|^{2}(y) \leq\left(A \nabla u+B \nabla^{\perp} u\right) \cdot\left(P^{T} \nabla u\right)(y) \\
& =\left(A \nabla u+B \nabla^{\perp} u\right) \cdot\left[\left(P^{T}(x)+\left(P^{T}-P^{T}(x)\right)\right) \nabla u\right](y),
\end{aligned}
$$

and therefore, by (4-5) and (5-3),

$$
\begin{aligned}
\left(\nabla^{\perp} \eta+\nabla \zeta\right) \cdot\left(P^{T}(x) \nabla u\right) & (y) \\
& =\left(A \nabla u+B \nabla^{\perp} u\right) \cdot\left(P^{T}(x) \nabla u\right)(y) \\
& \geq \frac{1}{2}|\nabla u|^{2}(y)-\left(A \nabla u+B \nabla^{\perp} u\right) \cdot\left[\left(P^{T}-P^{T}(x)\right) \nabla u\right](y) \geq \frac{1}{4}|\nabla u|^{2}(y) .
\end{aligned}
$$

Now we choose a function

$$
\phi \in C_{0}^{\infty}\left(B_{1}\right) \text { with } \phi \geq 0, \quad \operatorname{spt}(\phi) \subseteq B_{1 / 2}, \quad \phi=2 \text { on } B_{3 / 8}, \quad \text { and } \int_{B_{1}} \phi d x=1 .
$$

Moreover, we additionally assume that $\|\nabla \phi\|_{L^{\infty}\left(B_{1}\right)} \leq 100$. Using (4-3) and (5-5), one verifies directly that (by Definition 2.8)

$$
\begin{aligned}
\left\||\nabla u|^{2}\right\|_{h^{1}\left(B_{1}\right)} & =\int_{B_{1}} \sup _{0<t<1-|x|} \phi_{t} *|\nabla u|^{2} d x \\
& \leq 4 \int_{B_{1}} \sup _{0<t<1-|x|} \phi_{t} *\left(\left(\nabla^{\perp} \eta+\nabla \zeta\right) \cdot\left(P^{T}(x) \nabla u\right)\right) d x \\
& \left.=4 \int_{B_{1} 0<t<1-|x|} \sup _{t} \phi_{t} *\left(P^{T}(x)\right)_{i j}\left(\nabla^{\perp} \eta^{i} \cdot \nabla u^{j}+\nabla \zeta^{i} \cdot \nabla u^{j}\right)\right] d x \\
& \leq C \sum_{i, j=1}^{n}\left(\left\|\nabla^{\perp} \eta^{i} \cdot \nabla u^{j}\right\|_{h^{1}\left(B_{1}\right)}+\left\|\nabla \zeta^{i}\right\|_{W^{1,2}\left(B_{1}\right)}\left\|\nabla u^{j}\right\|_{L^{2}\left(B_{1}\right)}\right) \\
& \leq C\left\|\nabla^{\perp} \eta\right\|_{L^{2}\left(B_{1}\right)}\|\nabla u\|_{L^{2}\left(B_{1}\right)}+C \sqrt{\varepsilon_{0}}\left\|u_{t}\right\|_{L^{2}\left(B_{1}\right)} \leq C \varepsilon_{0},
\end{aligned}
$$

where we have used the relations

(1) $\nabla^{\perp} \eta^{i} \cdot \nabla u^{j} \in h^{1}\left(B_{1}\right)$ and $\left\|\nabla^{\perp} \eta^{i} \cdot \nabla u^{j}\right\|_{h^{1}\left(B_{1}\right)} \leq C\|\nabla \eta\|_{L^{2}\left(B_{1}\right)}\|\nabla u\|_{L^{2}\left(B_{1}\right)}$ for all $i, j=1,2, \ldots, n$;

(2) $\left\|\nabla \zeta^{i} \cdot \nabla u^{j}\right\|_{L^{p}\left(B_{1}\right)} \leq C\left\|\nabla \zeta^{i}\right\|_{W^{1,2}\left(B_{1}\right)}\left\|\nabla u^{j}\right\|_{L^{2}\left(B_{1}\right)}$ for any $1<p<2$ and $\|f\|_{h^{1}\left(B_{1}\right)} \leq C\|f\|_{L^{p}\left(B_{1}\right)}$ for any $p>1$. 
To see (1), we first extend

$$
\eta^{i}-\frac{1}{\left|B_{1}\right|} \int_{B_{1}} \eta^{i} \quad \text { and } \quad u^{j}-\frac{1}{\left|B_{1}\right|} \int_{B_{1}} u^{j}
$$

from $B_{1}$ to $\mathbb{R}^{2}$, which yields the existence of $\tilde{\eta}^{i}, \tilde{u}^{j} \in W_{c}^{1,2}\left(\mathbb{R}^{2}\right)$ such that

$$
\int_{\mathbb{R}^{2}}\left|\nabla \tilde{\eta}^{i}\right|^{2} \leq C \int_{B_{1}}\left|\nabla \eta^{i}\right|^{2} \quad \text { and } \quad \int_{\mathbb{R}^{2}}\left|\nabla \tilde{u}^{j}\right|^{2} \leq C \int_{B_{1}}\left|\nabla u^{j}\right|^{2}
$$

and

$$
\nabla \tilde{\eta}^{i}=\nabla \eta^{i} \quad \text { and } \quad \nabla \tilde{u}^{j}=\nabla u^{j} \quad \text { a.e. in } B_{1}
$$

Then, by the results of [Coifman et al. 1993], we know that

$$
\begin{aligned}
\left\|\nabla^{\perp} \tilde{\eta}^{i} \cdot \nabla \tilde{u}^{j}\right\|_{\mathcal{H}^{1}\left(\mathbb{R}^{2}\right)}: & =\int_{\mathbb{R}^{2}} \sup _{\phi \in \mathscr{T}} \sup _{t>0}\left|\int_{B_{t}(x)} \frac{1}{t^{2}} \phi\left(\frac{x-y}{t}\right)\left(\nabla^{\perp} \tilde{\eta}^{i} \cdot \nabla \tilde{u}^{j}\right)(y) d y\right| d x \\
& \leq C\left\|\nabla \tilde{\eta}^{i}\right\|_{L^{2}\left(\mathbb{R}^{2}\right)}\left\|\nabla \tilde{u}^{j}\right\|_{L^{2}\left(\mathbb{R}^{2}\right)} \leq C\|\nabla \eta\|_{L^{2}\left(B_{1}\right)}\|\nabla u\|_{L^{2}\left(B_{1}\right)},
\end{aligned}
$$

where $\mathscr{T}=\left\{\phi \in C^{\infty}\left(\mathbb{R}^{2}\right): \operatorname{spt}(\phi) \subset B_{1}\right.$ and $\left.\|\nabla \phi\|_{L^{\infty}} \leq 100\right\}$. By (5-8), (5-9), and Definition 2.8, it is clear that

$$
\begin{aligned}
\left\|\nabla^{\perp} \eta^{i} \cdot \nabla u^{j}\right\|_{h^{1}\left(B_{1}\right)} & =\left\|\nabla^{\perp} \tilde{\eta}^{i} \cdot \nabla \tilde{u}^{j}\right\|_{h^{1}\left(B_{1}\right)} \\
& \leq\left\|\nabla^{\perp} \tilde{\eta}^{i} \cdot \nabla \tilde{u}^{j}\right\|_{\mathscr{H}^{1}\left(\mathbb{R}^{2}\right)} \leq C\|\nabla \eta\|_{L^{2}\left(B_{1}\right)}\|\nabla u\|_{L^{2}\left(B_{1}\right)} .
\end{aligned}
$$

This completes the proof of the lemma.

Now, since $u(x, t) \in W^{1,2} \cap C^{\infty}\left(B_{1} \times[1, \infty), \mathcal{N}\right)$ and the energy $E(u(\cdot, t))$ is nonincreasing along the flow as shown in (1-6), there exists $T_{0} \geq 1$ such that

$$
\left\|u_{t}\left(T_{0}\right)\right\|_{L^{2}\left(B_{1}\right)}<\sqrt{\varepsilon_{0}} .
$$

Then by Lemma 5.1 we know that $\left|\nabla u\left(T_{0}\right)\right|^{2} \in h^{1}\left(B_{1}\right)$ with estimate

$$
\left\|\left|\nabla u\left(T_{0}\right)\right|^{2}\right\|_{h^{1}\left(B_{1}\right)} \leq C \varepsilon_{0} .
$$

Therefore, in view of Lemma 5.1, in order to validate the global estimate (2-21) we are left to show

$$
\left\|u_{t}\left(t_{0}\right)\right\|_{L^{2}\left(B_{1}\right)}<\sqrt{\varepsilon_{0}} \text { for all } t_{0} \geq T_{0}
$$

We will next show this is indeed the case.

Lemma 5.3. Let $u(x, t)$ be as in Theorem 1.6. Then there exists $T_{0}>0$ such that

$$
\left\|u_{t}\left(t_{0}\right)\right\|_{L^{2}\left(B_{1}\right)}<\sqrt{\varepsilon_{0}} \text { for all } t_{0} \geq T_{0} .
$$

Proof. Let $T_{0} \geq 1$ be as in (5-11), so $\left\|u_{t}\left(T_{0}\right)\right\|_{L^{2}\left(B_{1}\right)}<\sqrt{\varepsilon_{0}}$. Since $u(x, t) \in W^{1,2} \cap C^{\infty}\left(B_{1} \times[1, \infty), \mathcal{N}\right)$, and by the continuity of $\int_{B_{1}}\left|u_{t}(t)\right|^{2}$ in $t$, there exists $\delta=\delta\left(T_{0}, \varepsilon_{0}\right)>0$ such that, for any $t_{0} \in\left[T_{0}, T_{0}+\delta\right]$, we have

$$
\left\|u_{t}\left(t_{0}\right)\right\|_{L^{2}\left(B_{1}\right)}<2 \sqrt{\varepsilon_{0}}
$$


Therefore, by our previous arguments (especially Theorem 2.6, Lemma 5.1 and (5-12) with $T_{0}$ replaced by $\left.t_{0}\right)$, Lemma 2.5 applies to any subinterval of $\left[T_{0}, T_{0}+\delta\right]$ and yields

$$
\int_{B_{1}}\left|u_{t}\left(t_{2}\right)\right|^{2} \leq \int_{B_{1}}\left|u_{t}\left(t_{1}\right)\right|^{2} \text { for any } t_{1}, t_{2} \text { such that } T_{0} \leq t_{1}<t_{2} \leq T_{0}+\delta .
$$

This shows, instead of (5-15), for any $t_{0} \in\left[T_{0}, T_{0}+\delta\right]$, we have

$$
\left\|u_{t}\left(t_{0}\right)\right\|_{L^{2}\left(B_{1}\right)} \leq\left\|u_{t}\left(T_{0}\right)\right\|_{L^{2}\left(B_{1}\right)}<\sqrt{\varepsilon_{0}} .
$$

We can then continue and iterate this process beyond $T_{0}+\delta$ and we see that $\int_{B_{1}}\left|u_{t}(t)\right|^{2}$ is indeed nonincreasing along the flow after $T_{0}$.

This completes the validation of (2-21) and therefore the assumptions in Lemmas 2.4 and 2.5 in view of Theorem 2.6, finishing the proof of our main Theorem 1.6 as shown in Section 2.

\section{Appendix: A proof of Theorem 2.6}

Proof. The idea of the proof follows [Semmes 1994, Proposition 1.68]. Since the Green's function of $\Delta$ on $B_{1}$ is given by $(1 /(2 \pi)) \ln |x|$ for $x \in B_{1}$, we can write

$$
\psi(x)=\frac{1}{2 \pi} \int_{B_{1}} f(y)\left(\ln |x-y|-\ln \left(\left|\frac{x}{|x|}-\right| x|y|\right)\right) d y .
$$

Let $\theta \in C_{0}^{\infty}\left(B_{1}\right)$ be a smooth bump function such that $0 \leq \theta \leq 1, \theta=1$ in $B_{1 / 16}$ and $\operatorname{spt}(\theta) \subset B_{1 / 8}$. For $x \in B_{1}$, we define

$$
l_{x}(y):=\sum_{j=0}^{\infty} \theta\left(2^{j}(1-|x|)^{-1}(x-y)\right) \quad \text { for } y \in B_{1} .
$$

We claim that, for any $x, y \in B_{1}$,

$$
-20 \ln 2 \leq \ln |x-y|-\ln \left(\left|\frac{x}{|x|}-\right| x|y|\right)+l_{x}(y) \ln 2 \leq 20 \ln 2 .
$$

To see this, it is clear that, for $x, y \in B_{1}$ such that

$$
2^{-k} \leq|x-y| \leq 2^{-k+1}, \quad k \in \mathbb{N}_{0},
$$

we have

$$
-k \ln 2 \leq \ln |x-y| \leq(-k+1) \ln 2 .
$$

Now note that

$$
1-|x|-|x-y| \leq 1-|x|+|x|-|y|=1-|y| \leq 1-|x|+|x-y|,
$$

and therefore, for $x \in B_{1-2^{-i-1}} \backslash B_{1-2^{-i}}$, that is, $1-|x| \in\left[2^{-i-1}, 2^{-i}\right], i \in \mathbb{N}_{0}$ (with $\bar{B}_{0}=\varnothing$ ), and any $y \in B_{1}$ satisfying (A-4), we have

$$
1-|y| \in \begin{cases}{\left[2^{-i-1}-2^{-k+1}, 2^{-i}+2^{-k+1}\right]} & \text { if } k \geq i+4, \\ {\left[0,2^{-i}+2^{-k+1}\right]} & \text { if } k \leq i+3 .\end{cases}
$$


We also have

$$
\begin{aligned}
0 \leq(1-|x|)(1-|y|) & \leq\left(1-|x|^{2}\right)\left(1-|y|^{2}\right) \\
& =\left|\frac{x}{|x|}-\right| x|y|^{2}-|x-y|^{2} \leq 2^{2}(1-|x|)(1-|y|),
\end{aligned}
$$

and thus

$$
\left|\frac{x}{|x|}-\right| x|y|^{2}-|x-y|^{2} \in \begin{cases}{\left[2^{-2 i-2}-2^{-i-k}, 2^{-2 i+2}+2^{-i-k+3}\right]} & \text { if } k \geq i+4 \\ {\left[0,2^{-2 i+2}+2^{-i-k+3}\right]} & \text { if } k \leq i+3\end{cases}
$$

Combining this with (A-4), we get

$$
\left|\frac{x}{|x|}-\right| x|y|^{2} \in \begin{cases}{\left[2^{-2 i-2}-2^{-i-k}+2^{-2 k}, 2^{-2 i+2}+2^{-i-k+3}+2^{-2 k+2}\right]} & \text { if } k \geq i+4, \\ {\left[2^{-2 k}, 2^{-2 i+2}+2^{-i-k+3}+2^{-2 k+2}\right]} & \text { if } k \leq i+3 .\end{cases}
$$

Now, using the facts that for $k \geq i+4$ we have

$$
2^{-2 i-2}-2^{-i-k}+2^{-2 k} \geq 2^{-2 i-4} \text { and } 2^{-2 i+2}+2^{-i-k+3}+2^{-2 k+2} \leq 2^{-2 i+4}
$$

and for $k \leq i+3$ we have

$$
2^{-2 i+2}+2^{-i-k+3}+2^{-2 k+2} \leq 2^{-2 k+10},
$$

we arrive at

$$
\left|\frac{x}{|x|}-\right| x|y|^{2} \in \begin{cases}{\left[2^{-2 i-4}, 2^{-2 i+4}\right]} & \text { if } k \geq i+4 \\ {\left[2^{-2 k}, 2^{-2 k+10}\right]} & \text { if } k \leq i+3\end{cases}
$$

and hence

$$
-\ln \left|\frac{x}{|x|}-\right| x|y| \in \begin{cases}{[(i-2) \ln 2,(i+2) \ln 2]} & \text { if } k \geq i+4, \\ {[(k-5) \ln 2, k \ln 2]} & \text { if } k \leq i+3 .\end{cases}
$$

Combining (A-5) and (A-7), we get

$$
\ln |x-y|-\ln \left|\frac{x}{|x|}-\right| x|y| \in \begin{cases}{[(-k+i-2) \ln 2,(-k+i+3) \ln 2]} & \text { if } k \geq i+4, \\ {[-5 \ln 2, \ln 2] \quad(\text { in fact, }[-5 \ln 2,0])} & \text { if } k \leq i+3\end{cases}
$$

for any $x \in B_{1-2^{-i-1}} \backslash B_{1-2^{-i}}, i \geq 0$, and any $y \in B_{1}$ satisfying (A-4) for some $k \geq 0$.

Now, for any $x \in B_{1-2^{-i-1}} \backslash B_{1-2^{-i}}, i \geq 0$, and any $y \in B_{1}$ satisfying (A-4), since $0 \leq \theta \leq 1, \theta=1$ in $B_{1 / 16}$, and $\operatorname{spt}(\theta) \subset B_{1 / 8}$, we get that, for any $j \geq 0$,

$$
\theta\left(2^{j}(1-|x|)^{-1}(x-y)\right)=0 \text { for }|x-y| \geq 2^{-j-3}(1-|x|) \in\left[2^{-j-i-4}, 2^{-j-i-3}\right]
$$

and

$$
\theta\left(2^{j}(1-|x|)^{-1}(x-y)\right)=1 \quad \text { for }|x-y| \leq 2^{-j-4}(1-|x|) \in\left[2^{-j-i-5}, 2^{-j-i-4}\right] .
$$

Combining with (A-4), we obtain

$$
\theta\left(2^{j}(1-|x|)^{-1}(x-y)\right)=0 \text { for } j \geq k-i-3
$$

and

$$
\theta\left(2^{j}(1-|x|)^{-1}(x-y)\right)=1 \quad \text { if } k-1 \geq j+i+5 \quad(\text { that is } j \leq k-i-6) .
$$


Hence, for any $x \in B_{1-2^{-i-1}} \backslash B_{1-2^{-i}}, i \geq 0$ and any $y \in B_{1}$ such that $2^{-k} \leq|x-y| \leq 2^{-k+1}$ for some $k=0,1,2, \ldots,(\mathrm{A}-2),(\mathrm{A}-9)$, and (A-10) imply

$$
\begin{cases}k-i-10 \leq l_{x}(y) \leq k-i+10 & \text { if } k \geq i+4 \\ l_{x}(y)=0 & \text { if } k \leq i+3 .\end{cases}
$$

Combining (A-8) and (A-11) gives (A-3).

Therefore, in order to obtain the $L^{\infty}$-bound of $\psi$ on $B_{1}$ as in (2-18), it suffices to bound $\int_{B_{1}} f(y) l_{x}(y) d y$, since we have (A-1), (A-3), and $\|f\|_{L^{1}\left(B_{1}\right)} \leq\|f\|_{h^{1}\left(B_{1}\right)}$.

In order to bound $\int_{B_{1}} f(y) l_{x}(y) d y$, we next claim that, for any $x \in B_{1}, j \geq 0$, and $z \in B_{2^{-j-4}(1-|x|)}(x)$, we have

$$
\int_{B_{1}} f(y) 2^{2 j+2}(1-|x|)^{-2} \theta\left(2^{j}(1-|x|)^{-1}(x-y)\right) d y \leq \int_{B_{t}(z)} \frac{1}{t^{2}} \phi\left(\frac{z-y}{t}\right) f(y) d y,
$$

where

$$
t=2^{-j-1}(1-|x|)
$$

and $\phi$ is a nonnegative Schwartz function as in (5-6). To see (A-12), we first note that since $\operatorname{spt}(\theta) \subset B_{1 / 8}$, we have, for any $x \in B_{1}$ and $j \geq 0$,

$$
\begin{aligned}
\int_{B_{1}} f(y) 2^{2 j+2}(1-|x|)^{-2} \theta & \left(2^{j}(1-|x|)^{-1}(x-y)\right) d y \\
= & \int_{B_{2^{-j-3}(1-|x|)}(x)} f(y) 2^{2 j+2}(1-|x|)^{-2} \theta\left(2^{j}(1-|x|)^{-1}(x-y)\right) d y .
\end{aligned}
$$

Now since

$$
\frac{3}{8}\left(2^{-j-1}\right)=2^{-j-4}+2^{-j-3}
$$

and

$$
2^{-j-4}+2^{-j-1}=\frac{9}{16} 2^{-j}<1
$$

for any $j \geq 0$, we see that, for any $z \in B_{2^{-j-4}(1-|x|)}(x)$,

$$
B_{2^{-j-3}(1-|x|)}(x) \subseteq B_{3 t / 8}(z) \subset B_{t}(z)=B_{2^{-j-1}(1-|x|)}(z) \subseteq B_{1}
$$

Using the relations $f \geq 0,0 \leq \theta \leq 1, \phi \geq 0$, and the fact that $\phi=2$ on $B_{3 / 8}$, we conclude

$$
\begin{aligned}
\int_{B_{2^{-j-3}(1-|x|)}(x)} f(y) 2^{2 j+2}(1 & -|x|)^{-2} \theta\left(2^{j}(1-|x|)^{-1}(x-y)\right) d y \\
& \leq \int_{B_{2^{-j-3}(1-|x|)}(x)} f(y) 2^{2 j+2}(1-|x|)^{-2} d y \leq \int_{B_{3 t / 8}(z)} f(y) 2^{2 j+2}(1-|x|)^{-2} d y \\
& \leq \int_{B_{t}(z)} f(y) 2^{2 j+2}(1-|x|)^{-2} \phi\left(\frac{z-y}{t}\right) d y=\int_{B_{t}(z)} \frac{1}{t^{2}} \phi\left(\frac{z-y}{t}\right) f(y) d y
\end{aligned}
$$


Combining this with (A-13) gives (A-12). Therefore, by (A-12) and the definition (2-19) of the radial maximal function $f^{*}$, for any $x \in B_{1}$ and $j \geq 0$, we have

$$
\left|\int_{B_{1}} f(y) \theta\left(2^{j}(1-|x|)^{-1}(x-y)\right) d y\right| \leq 2^{-2 j-2}(1-|x|)^{2} \inf _{z \in B_{2^{-j-4}(1-|x|)}(x)} f^{*}(z) .
$$

Therefore, by (A-2), for any $x \in B_{1}$, we have

$$
\begin{aligned}
\left|\int_{B_{1}} f(y) l_{x}(y) d y\right| & \leq \sum_{j=0}^{\infty}\left|\int_{B_{1}} f(y) \theta\left(2^{j}(1-|x|)^{-1}(x-y)\right) d y\right| \\
& \leq \sum_{j=0}^{\infty} 2^{-2 j-2}(1-|x|)^{2} \inf _{z \in B_{2^{-j-4}(1-|x|)}(x)} f^{*}(z) \\
& \leq \frac{2^{8}}{3 \pi} \sum_{j=0}^{\infty} \int_{B_{2^{-j-4}(1-|x|)}(x) \backslash B_{2^{-j-5}(1-|x|)}(x)} f^{*}(z) d z \\
& \leq \frac{2^{8}}{3 \pi} \int_{B_{1}} f^{*}(z) d z \leq \frac{2^{8}}{3 \pi}\|f\|_{h^{1}\left(B_{1}\right)} .
\end{aligned}
$$

Combining (A-6), (A-3), and (A-15) yields (using $\left.\|f\|_{L^{1}\left(B_{1}\right)} \leq\|f\|_{h^{1}\left(B_{1}\right)}\right)$

$$
|\psi(x)|=-\frac{1}{2 \pi} \int_{B_{1}} f(y)\left(\ln |x-y|-\ln \left|\frac{x}{|x|}-\right| x|y|\right) d y \leq C\|f\|_{h^{1}\left(B_{1}\right)} .
$$

This gives the desired $L^{\infty}$-bound of $\psi$ on $B_{1}$. The $L^{2}$-estimate for $\nabla \psi$ simply follows from an integration by parts argument.

\section{Acknowledgement}

The author thanks Bill Minicozzi for his continued guidance and support. The author also thanks his collaborator Tobias Lamm for all the valuable discussions.

\section{References}

[Brezis and Coron 1983] H. Brezis and J.-M. Coron, "Large solutions for harmonic maps in two dimensions", Comm. Math. Phys. 92:2 (1983), 203-215. MR 85a:58022 Zbl 0532.58006

[Brezis and Coron 1984] H. Brezis and J.-M. Coron, "Multiple solutions of $H$-systems and Rellich's conjecture", Comm. Pure Appl. Math. 37:2 (1984), 149-187. MR 85i:53010 Zbl 0537.49022

[Chang 1989] K.-C. Chang, "Heat flow and boundary value problem for harmonic maps", Ann. Inst. H. Poincaré Anal. Non Linéaire 6:5 (1989), 363-395. MR 90i:58037 Zbl 0687.58004

[Chang et al. 1993] D.-C. Chang, S. G. Krantz, and E. M. Stein, " $H^{p}$ theory on a smooth domain in $\mathbb{R}^{N}$ and elliptic boundary value problems”, J. Funct. Anal. 114:2 (1993), 286-347. MR 94j:46032 Zbl 0804.35027

[Choné 1995] P. Choné, "A regularity result for critical points of conformally invariant functionals", Potential Anal. 4:3 (1995), 269-296. MR 96e:58041 Zbl 0833.53010

[Coifman et al. 1993] R. Coifman, P.-L. Lions, Y. Meyer, and S. Semmes, "Compensated compactness and Hardy spaces", J. Math. Pures Appl. (9) 72:3 (1993), 247-286. MR 95d:46033 Zbl 0864.42009 
[Colding and Minicozzi 2008a] T. H. Colding and W. P. Minicozzi, II, "Width and finite extinction time of Ricci flow", Geom. Topol. 12:5 (2008), 2537-2586. MR 2009k:53166 Zbl 1161.53352

[Colding and Minicozzi 2008b] T. H. Colding and W. P. Minicozzi, II, "Width and mean curvature flow", Geom. Topol. 12:5 (2008), 2517-2535. MR 2009k:53165 Zbl 1165.53363

[Eells and Sampson 1964] J. Eells, Jr. and J. H. Sampson, "Harmonic mappings of Riemannian manifolds", Amer. J. Math. 86 (1964), 109-160. MR 29\#1603 Zbl 0122.40102

[Freire 1995] A. Freire, "Uniqueness for the harmonic map flow from surfaces to general targets", Comment. Math. Helv. 70:2 (1995), 310-338. MR 96f:58045 Zbl 0831.58018

[Freire 1996] A. Freire, "Correction to: "Uniqueness for the harmonic map flow from surfaces to general targets" [Comment. Math. Helv. 70 (1995), no. 2, 310-338; MR1324632 (96f:58045)]”, Comment. Math. Helv. 71:2 (1996), 330-337. MR 97c:58032 Zbl 0851.58011

[Hamilton 1975] R. S. Hamilton, Harmonic maps of manifolds with boundary, Lecture Notes in Mathematics 471, Springer, Berlin, 1975. MR 58 \#2872 Zbl 0308.35003

[Hartman 1967] P. Hartman, “On homotopic harmonic maps”, Canad. J. Math. 19 (1967), 673-687. MR 35 \#4856 Zbl 0155. 49705

[Hélein 2002] F. Hélein, Harmonic maps, conservation laws and moving frames, 2nd ed., Cambridge Tracts in Mathematics 150, Cambridge University Press, 2002. MR 2003g:58024 Zbl 1010.58010

[Iwaniec and Martin 2001] T. Iwaniec and G. Martin, Geometric function theory and non-linear analysis, The Clarendon Press Oxford University Press, New York, 2001. MR 2003c:30001 Zbl 1045.30011

[Jost 1984] J. Jost, "The Dirichlet problem for harmonic maps from a surface with boundary onto a 2-sphere with nonconstant boundary values", J. Differential Geom. 19:2 (1984), 393-401. MR 86b:58031 Zbl 0551.58012

[Lamm and Lin 2013] T. Lamm and L. Lin, "Estimates for the energy density of critical points of a class of conformally invariant variational problems", Adv. Calc. Var. 6:4 (2013), 391-413. Zbl 06224215 arXiv 1202.5758

[Lieberman 1996] G. M. Lieberman, Second order parabolic differential equations, World Scientific, River Edge, NJ, 1996. MR 98k:35003 Zbl 0884.35001

[Miyachi 1990] A. Miyachi, “ $H^{p}$ spaces over open subsets of $\mathbf{R}^{n}$ ”, Studia Math. 95:3 (1990), 205-228. MR 91m:42022 Zbl 0716.42017

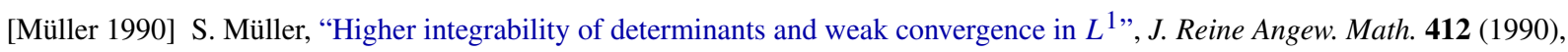
20-34. MR 92b:49026 Zbl 0713.49004

[Qing 1995] J. Qing, "On singularities of the heat flow for harmonic maps from surfaces into spheres", Comm. Anal. Geom. 3:1-2 (1995), 297-315. MR 97c:58154 Zbl 0868.58021

[Rivière 2007] T. Rivière, "Conservation laws for conformally invariant variational problems", Invent. Math. 168:1 (2007), 1-22. MR 2008d:58010 Zbl 1428.58010

[Rivière 2008] T. Rivière, "Analysis aspects of Willmore surfaces", Invent. Math. 174:1 (2008), 1-45. MR 2009k:53154 Zbl 1155.53031

[Rivière 2011] T. Rivière, "The role of integrability by compensation in conformal geometric analysis", pp. 93-127 in Analytic aspects of problems in Riemannian geometry: elliptic PDEs, solitons and computer imaging, edited by P. Baird et al., Sémin. Congr. 22, Soc. Math. France, Paris, 2011. MR 3060451

[Rivière 2012] T. Rivière, “Conformally invariant variational problems”, preprint, 2012. arXiv 1206.2116

[Schikorra 2010] A. Schikorra, "A remark on gauge transformations and the moving frame method.", Ann. Inst. Henri Poincaré, Anal. Non Linéaire 27:2 (2010), 503-515. Zbl 1187.35054

[Semmes 1994] S. Semmes, "A primer on Hardy spaces, and some remarks on a theorem of Evans and Müller", Comm. Partial Differential Equations 19:1-2 (1994), 277-319. MR 94j:46038 Zbl 0836.35030

[Simon 1983] L. Simon, "Asymptotics for a class of nonlinear evolution equations, with applications to geometric problems", Ann. of Math. (2) 118:3 (1983), 525-571. MR 85b:58121 Zbl 0549.35071

[Struwe 1985] M. Struwe, "On the evolution of harmonic mappings of Riemannian surfaces", Comment. Math. Helv. 60:4 (1985), 558-581. MR 87e:58056 Zbl 0595.58013 
[Tartar 1985] L. Tartar, "Remarks on oscillations and Stokes' equation", pp. 24-31 in Macroscopic modelling of turbulent flows (Nice, 1984), edited by U. Frisch et al., Lecture Notes in Phys. 230, Springer, Berlin, 1985. MR 815930 Zbl 0611.76042

[Topping 1997] P. M. Topping, "Rigidity in the harmonic map heat flow", J. Differential Geom. 45:3 (1997), 593-610. MR 99d:58050 Zbl 0955.58013

[Uhlenbeck 1982] K. K. Uhlenbeck, "Connections with $L^{p}$ bounds on curvature”, Comm. Math. Phys. 83:1 (1982), 31-42. MR 83e:53035 Zbl 0499.58019

[Wang 2012] L. Wang, "Harmonic map heat flow with rough boundary data", Trans. Amer. Math. Soc. 364:10 (2012), 5265-5283. MR 2931329

[Wente 1969] H. C. Wente, "An existence theorem for surfaces of constant mean curvature", J. Math. Anal. Appl. 26 (1969), 318-344. MR 39 \#4788 Zbl 0181.11501

Received 23 Jul 2012. Accepted 22 Aug 2013.

LONGZHI LIN: lzlin@math.rutgers .edu

Department of Mathematics, Rutgers University, 110 Frelinghuysen Road, Piscataway, New Jersey 08854-8019, United States 


\title{
Analysis \& PDE
}

\author{
msp.org/apde
}

\section{EDITORS}

EDITOR-IN-CHIEF

\author{
Maciej Zworski \\ zworski@math.berkeley.edu \\ University of California \\ Berkeley, USA
}

BOARD OF EDITORS

Nicolas Burq Université Paris-Sud 11, France

nicolas.burq@math.u-psud.fr

Sun-Yung Alice Chang Princeton University, USA

chang@math.princeton.edu

Michael Christ University of California, Berkeley, USA

mchrist@math.berkeley.edu

Charles Fefferman Princeton University, USA

cf@math.princeton.edu

Ursula Hamenstaedt Universität Bonn, Germany

ursula@math.uni-bonn.de

Vaughan Jones U.C. Berkeley \& Vanderbilt University vaughan.f.jones@vanderbilt.edu

Herbert Koch Universität Bonn, Germany koch@math.uni-bonn.de

Izabella Laba University of British Columbia, Canada ilaba@math.ubc.ca

Gilles Lebeau Université de Nice Sophia Antipolis, France lebeau@unice.fr

László Lempert Purdue University, USA lempert@math.purdue.edu

Richard B. Melrose Massachussets Institute of Technology, USA rbm@math.mit.edu

Frank Merle Université de Cergy-Pontoise, France Frank.Merle@u-cergy.fr

William Minicozzi II Johns Hopkins University, USA minicozz@math.jhu.edu

Werner Müller Universität Bonn, Germany mueller@math.uni-bonn.de
Yuval Peres

Gilles Pisier

Tristan Rivière

Igor Rodnianski

Wilhelm Schlag

Sylvia Serfaty

Yum-Tong Siu

Terence Tao

Michael E. Taylor

Gunther Uhlmann

András Vasy

Dan Virgil Voiculescu

Steven Zelditch
University of California, Berkeley, USA

peres@stat.berkeley.edu

Texas A\&M University, and Paris 6

pisier@math.tamu.edu

ETH, Switzerland

riviere@math.ethz.ch

Princeton University, USA

irod@math.princeton.edu

University of Chicago, USA

schlag@math.uchicago.edu

New York University, USA

serfaty@cims.nyu.edu

Harvard University, USA

siu@math.harvard.edu

University of California, Los Angeles, USA

tao@math.ucla.edu

Univ. of North Carolina, Chapel Hill, USA

met@math.unc.edu

University of Washington, USA

gunther@math.washington.edu

Stanford University, USA

andras@math.stanford.edu

University of California, Berkeley, USA

dvv@math.berkeley.edu

Northwestern University, USA

zelditch@math.northwestern.edu

\section{PRODUCTION}

production@msp.org

Silvio Levy, Scientific Editor

See inside back cover or msp.org/apde for submission instructions.

The subscription price for 2013 is US \$160/year for the electronic version, and \$310/year ( $\$ 35$, if shipping outside the US) for print and electronic. Subscriptions, requests for back issues from the last three years and changes of subscribers address should be sent to MSP.

Analysis \& PDE (ISSN 1948-206X electronic, 2157-5045 printed) at Mathematical Sciences Publishers, 798 Evans Hall \#3840, c/o University of California, Berkeley, CA 94720-3840, is published continuously online. Periodical rate postage paid at Berkeley, CA 94704, and additional mailing offices.

APDE peer review and production are managed by EditFLOW ${ }^{\circledR}$ from Mathematical Sciences Publishers.

PUBLISHED BY

- mathematical sciences publishers

nonprofit scientific publishing

http://msp.org/

(C) 2013 Mathematical Sciences Publishers 


\section{ANALYSIS \& PDE \\ Volume $6 \quad$ No. $8 \quad 2013$}

$L^{p}$ and Schauder estimates for nonvariational operators structured on Hörmander vector fields 1793 with drift

Marco Bramanti and MaOchun ZhU

Strichartz estimates for Schrödinger equations with variable coefficients and unbounded po- 1857 tentials

HARUYA MIZUTANI

Uniformity of harmonic map heat flow at infinite time

1899

LONGZHI LIN

A rotational approach to triple point obstructions

1923

NOAH SNYDER

On the energy subcritical, nonlinear wave equation in $\mathbb{R}^{3}$ with radial data RUIPENG SHEN

Global well-posedness for the nonlinear Schrödinger equation with derivative in energy space 1989 YIFEI WU

The Calderón problem with partial data on manifolds and applications

2003

CARlos Kenig and MikKo SAlo 